\title{
A Neural Network Underlying Circadian Entrainment and Photoperiodic Adjustment of Sleep and Activity in Drosophila
}

\author{
- Matthias Schlichting, ${ }^{1 *}$-Pamela Menegazzi, ${ }^{1 *}$ Katharine R. Lelito, ${ }^{2}{ }^{\circ}$ Zepeng Yao, ${ }^{2} \odot$ Edgar Buhl, ${ }^{3}$ \\ Elena Dalla Benetta, ${ }^{1}$ Andrew Bahle, ${ }^{2}$ Jennifer Denike, ${ }^{2}$ - James John Hodge, ${ }^{3}$ CCharlotte Helfrich-Förster, ${ }^{1}$ \\ and $\mathbb{D}$ Orie Thomas Shafer ${ }^{2}$ \\ ${ }^{1}$ Neurobiology and Genetics, Biocenter, University of Wuerzburg, 97074 Würzburg, Germany, ${ }^{2}$ Department of Molecular Cellular and Developmental \\ Biology, University of Michigan, Ann Arbor, Michigan 48109, and ${ }^{3}$ School of Physiology and Pharmacology, University of Bristol, Bristol BS8 1TH, \\ United Kingdom
}

A sensitivity of the circadian clock to light/dark cycles ensures that biological rhythms maintain optimal phase relationships with the external day. In animals, the circadian clock neuron network (CCNN) driving sleep/activity rhythms receives light input from multiple photoreceptors, but how these photoreceptors modulate CCNN components is not well understood. Here we show that the HofbauerBuchner eyelets differentially modulate two classes of ventral lateral neurons (LNvs) within the Drosophila CCNN. The eyelets antagonize Cryptochrome (CRY)- and compound-eye-based photoreception in the large LNvs while synergizing CRY-mediated photoreception in the small LNvs. Furthermore, we show that the large LNvs interact with subsets of "evening cells" to adjust the timing of the evening peak of activity in a day length-dependent manner. Our work identifies a peptidergic connection between the large LNvs and a group of evening cells that is critical for the seasonal adjustment of circadian rhythms.

Key words: circadian; entrainment; photoreception; pigment dispersing factor

\section{Significance Statement}

In animals, circadian clocks have evolved to orchestrate the timing of behavior and metabolism. Consistent timing requires the entrainment these clocks to the solar day, a process that is critical for an organism's health. Light cycles are the most important external cue for the entrainment of circadian clocks, and the circadian system uses multiple photoreceptors to link timekeeping to the light/dark cycle. How light information from these photorecptors is integrated into the circadian clock neuron network to support entrainment is not understood. Our results establish that input from the HB eyelets differentially impacts the physiology of neuronal subgroups. This input pathway, together with input from the compound eyes, precisely times the activity of flies under long summer days. Our results provide a mechanistic model of light transduction and integration into the circadian system, identifying new and unexpected network motifs within the circadian clock neuron network.

\section{Introduction}

Circadian clocks create an endogenous sense of time that is used to produce daily rhythms in physiology and behavior (Aschoff, 1981a). A defining characteristic of a circadian clock is a modest deviation of its endogenous period from the $24.0 \mathrm{~h}$ period of daily environmental change (Aschoff, 1981b). For example, the aver-
Received March 25, 2016; revised July 4, 2016; accepted July 9, 2016.

Author contributions: J.J.H., C.H.-F., and 0.T.S. designed research; M.S., P.M., K.R.L., Z.Y., E.D.B., A.B., and J.D. performed research; M.S., P.M., K.R.L., Z.Y., E.D.B., A.B., J.D., J.J.H., C.H.-F., and O.T.S. analyzed data; M.S., P.M., K.R.L., Z.Y., E.B., J.J.H., C.H.-F., and 0.T.S. wrote the paper.

This work was supported by National Institutes of Health (National Institute of Neurological Disorders and Stroke) Grants R00NS062953 and R01NS077933 to 0.T.S.; German Research Foundation Grants DFG F0207/10-3, SFB1047, and INST 93/784-1 and the European Community Sixth Framework Project EUCLOCK 018741 to C.H.-F.; Biotechnology and Biological Sciences Research Council Grant BB/J0127221/1 to J.J.H.; and BMBF (German Ministry for Education and Research) Hanns-Seidel-Foundation Excellence Grant to M.S. We thank Jae Park, Patrick Emery, Paul Hardin, Francois Rouyer, Paul Taghert, and Nick Glossop for sharing fly strains; Paul Hardin, Dick Nässel, Heinrich
Dircksen, and Takeshi Todo for providing antisera; Taishi Yoshii and Christiane Hermann-Luibl for characterization of the R78G02-GAL4 line; and Kate Abruzzi and Michael Rosbash for helpful comments on this manuscript.

The authors declare no competing financial interests.

${ }^{*}$ M.S. and P.M. contributed equally to this study.

Correspondence should be addressed to either of the following: Dr. Orie Thomas Shafer, Department of Molecular Cellular and Developmental Biology, University of Michigan, 830 N. University Avenue, Ann Arbor, MI 48109, E-mail: oshafer@umich.edu; or Dr. Charlotte Helfrich-Förster, Sanderring 2, 97074 Würzburg, Germany, E-mail: charlotte.foerster@biozentrum.uni-wuerzburg.de.

M. Schlichting's present address: Howard Hughes Medical Institute, Biology Department, Brandeis University, Waltham, MA 02453.

DO1:10.1523/JNEUROSCI.0992-16.2016

Copyright $\odot 2016$ the authors $\quad 0270-6474 / 16 / 369084-13 \$ 15.00 / 0$ 
age human clock has an endogenous period of $24 \mathrm{~h}$ and $11 \mathrm{~min}$ (Czeisler et al., 1999). Thus, to maintain a consistent phase relationship with the environment, the human clock must be sped up by 11 min every day. A sensitivity of the circadian clock to environmental time cues (zeitgebers) ensures that circadian clocks are adjusted daily to match the period of environmental change (Pittendrigh, 1981). This process, called entrainment, is fundamental to the proper daily timing of behavior and physiology (Roenneberg and Merrow, 2003). For most organisms, daily light/dark (LD) cycles are the most salient zeitgeber (Aschoff, 1981b).

Although most tissues express molecular circadian clocks in animals, the clock is required in small islands of neural tissue for the presence of sleep/activity rhythms and many other daily rhythms in physiology (Herzog, 2007). Within these islands, a circadian clock neuron network (CCNN) functions as the master circadian clock (Nitabach and Taghert, 2008; Welsh et al., 2010). Subsets of neurons within the CCNN receive resetting signals from photoreceptors, and physiological connections between these neurons and their clock neuron targets ensure light entrainment of the CCNN as a whole (Golombek and Rosenstein, 2010).

In both mammals and insects, the CCNN receives light input from multiple photoreceptor types. In Drosophila, the CCNN is entrained by photoreceptors in the compound eye, the ocelli, the Hofbauer-Buchner (HB) eyelets, and by subsets of clock neurons that express the blue light photoreceptor Cryptochrome (CRY) (Helfrich-Förster, 2002). Understanding how multiple light input pathways modulate the CCNN to ensure entrainment to the environmental LD cycle is critical for our understanding of the circadian system and its dysfunction when exposed to the unnatural light regimens accompanying much of modern life (Münch and Bromundt, 2012).

Here we investigate the physiological basis and circadian role of a long-suspected circadian light input pathway in Drosophila: the HB eyelets. These simple accessory eyes contain four photoreceptors located at the posterior edges of the compound eyes and project directly to the accessory medullae (AMe) (Hofbauer and Buchner, 1989; Helfrich-Förster et al., 2002; Malpel et al., 2002), neuropils that support circadian timekeeping in insects (Helfrich-Förster, 1998). In Drosophila, the AMe contain projections from ventral lateral neurons (LNvs), important components of the CCNN that express the neuropeptide pigment dispersing factor (PDF) (Helfrich-Förster, 1997, 1998), an output required for robust circadian rhythms in locomotor activity (Renn et al., 1999). The axon terminals of the HB eyelets terminate near PDF-positive LNv projections (HelfrichFörster et al., 2002; Malpel et al., 2002) and analysis of visual system and cry mutants reveals a role for the $\mathrm{HB}$ eyelet in the entrainment of locomotor rhythms to LD cycles (Helfrich-Förster et al., 2001, 2002; Veleri et al., 2007), but how the eyelets influence the CCNN to support light entrainment is not well understood.

Here we present evidence that this circadian light input pathway excites the small LNvs (s-LNvs) and acts to phase-dependently advance free-running rhythms in sleep/activity while inhibiting the large LNvs (1-LNvs). This work reveals that input from external photoreceptors differentially affects specific centers within the fly CCNN. Furthermore, we show that, under long summer-like days, the l-LNvs act to modulate subsets of so-called evening cells to delay the onset of evening activity. These results reveal a neural network underlying the photoperiodic adjustment of sleep and activity.

\section{Materials and Methods}

Transgenic strains. The following fly strains were used: For GFP Reconstitution Across Synaptic Partners (GRASP): LexAop-GFP-11; UAS-
GFP1-10 (Gordon and Scott, 2009); Pdf-LexA (Shang et al., 2008); Rh6-GAL4 (Sprecher and Desplan, 2008); R78G02-GAL4 (Bloomington Line BL40010). For expression analysis: UAS-cd8GFP (Siegmund and Korge, 2001); UAS-StingerGFP (Barolo et al., 2000); R78G02-GAL4 (BL40010). For live imaging experiments: Pdf(M)-Gal4 (Renn et al., 1999); UAS-GCaMP3.0 (Tian et al., 2009); UAS-P2X2 (Lima and Miesenböck, 2005); UAS-Epac1-camps(50A) (Shafer et al., 2008); LexAop-GCaMP3.0; LexAop-Epac1-camps (Yao et al., 2012); Rh6-GAL4. For locomotor activity experiments: UAS-TrpA1 (Hamada et al., 2008); $h d c^{J K 910}$ (Burg et al., 1993); Rh6-GAL4; UAS-Kir2.1 (Baines et al., 2001); UAS-pdfRNAi (BL25802); $h a n^{5304}$;UAS-PdfR (Hyun et al., 2005; Mertens et al., 2005); Pdf(M)-GAL4; R78G02-GAL4; R6-GAL4 (Helfrich-Förster et al., 2007); c929-GAL4 (Hewes et al., 2003); cry39-GAL4 (Picot et al., 2007); Clk856-GAL4 (Gummadova et al., 2009); Mai179-GAL4 (Grima et al., 2004); npf-GAL4 (Lee et al., 2006); clk4.1M-GAL4 (Zhang et al., 2010); wild-type CantonS. For electrophysiology, we used a Pdf-RFP (Ruben et al., 2012) construct. Male flies were used for all experiments.

GRASP and microscopy. To determine possible synapses between Rh6 cells and PDF neurons, we performed GRASP experiments and processed the brains with anti-PDF. A similar approach was used to visualize putative synaptic contacts between the PDF neurons and R78G02-GAL4expressing neurons.

Immunocytochemistry was performed as described by Menegazzi et al. (2013). We used the following primary antibodies: mouse anti-PDF (1:1000; Developmental Studies Hybridoma Bank, University of Iowa); chicken anti-GFP (1:2000; Abcam); rabbit anti-CRY (1:1000) (Yoshii et al., 2008); guinea pig anti-VRI (1:3000) (Glossop et al., 2003); rabbit anti-ion transport peptide (ITP; 1:1000) (Hermann-Luibl et al., 2014); mouse anti-GFP (1:1000; Sigma). Secondary antibodies were purchased from Invitrogen and used in a dilution of 1:200. Brains were mounted on glass slides using Vectashield mounting medium (Vector Laboratories).

We imaged brains using a Leica TCS SPE scanning confocal microscope (Leica Microsystems). We used 488, 555, and $635 \mathrm{~nm}$ laser diodes to excite GFP and the fluorophores of the secondary antibodies using confocal steps of $2 \mu \mathrm{m}$. The laser settings were kept constant within each experiment. All images were analyzed using the Fiji plug-in in ImageJ.

Live imaging. We performed live imaging experiments using an FV 1000 laser-scanning microscope (Olympus) and a $60 \times 1.1 \mathrm{~N} / \mathrm{A} \mathrm{W}$, FUMFL $\mathrm{N}$ objective (Olympus). We anesthetized flies over $\mathrm{CO}_{2}$ and dissected brains under HL3 saline (Stewart et al., 1994). We mounted brains on the bottom of $35 \mathrm{~mm}$ FALCON culture dishes (BD Biosciences) under a drop of HL3 saline within a Petri Dish Insert (BD Biosciences). We allowed brains to settle for 5-10 min before imaging. We maintained continuous HL3 perfusion while the regions of interest containing LNv somata or the HB eyelet nerve were selected using Olympus Fluoview software (Olympus). We performed $\mathrm{Ca}^{2+}$ imaging using the sensor GCaMP3.0, scanning brains with a $488 \mathrm{~nm}$ laser at $1 \mathrm{~Hz}$, and collecting GFP emission. We performed cAMP imaging using the FRET sensor Epac1-camps, scanning brains with a $440 \mathrm{~nm}$ laser at $1 \mathrm{~Hz}$, and collecting CFP and YFP emission. We processed GCaMP3.0 fluorescence and Epac1-camps inverse FRET (CFP/YFP) as previously described (Lelito and Shafer, 2012; Yao et al., 2012). GCaMP3.0 intensity values were filtered with a 10 point moving average. Intensity traces were then transformed to a percentage fluorescence change $\left(\Delta \mathrm{F} / \mathrm{F}_{0}\right)$ trace using the following equation: $\left(\left(\mathrm{F}_{\mathrm{n}}-\mathrm{F}_{0}\right) / \mathrm{F}_{0}\right) \times 100$, where $\mathrm{F}_{\mathrm{n}}$ is the intensity value at each point in time and $\mathrm{F}_{0}$ is the baseline fluorescence calculated as the average fluorescence intensity recorded during the first $10 \mathrm{~s}$ of imaging. Mean \pm SEM traces were created based on all GCaMP3.0 fluorescence traces for each neuronal class and treatment. The maximum increase in GCaMP3.0 fluorescence was determined for each filtered and normalized trace based on the entire duration of the recording. These values were then used to determine the mean maximum GCaMP3.0 fluorescence change for each treatment and neuron type. Epac1-camps recordings consisted of raw CFP and YFP values for each time-point. Spillover was corrected using the following equation: $\mathrm{YFP}_{\text {soc }}=\mathrm{YFP}-(\mathrm{CFP} \times 0.444)$, where $\mathrm{YFP}_{\text {soc }}$ is the spillover-corrected YFP intensity, YFP and CFP are the raw intensity values, and 0.444 is the proportion of CFP spillover into the YFP channel. FRET time courses were plotted as CFP/YFP ${ }_{\text {soc }}$, the so-called inverse FRET ratio, which was proportional to cAMP levels. Inverse FRET 
traces were filtered with a 10 point moving average, and the initial time-point for each trace was normalized to 1.0. These filtered and normalized inverse FRET ratio traces were averaged to create average traces for each treatment and neuron type and were expressed as percentage changes. The maximum change in inverse Epac1-camps FRET was determined for each individual trace from the duration of the recording and used to generate the mean maximum changes in inverse Epac1-camps FRET for each treatment and neuron type.

Each imaging experiment began with the acquisition of $30 \mathrm{~s}$ of baseline fluorescence. At $30 \mathrm{~s}$, we switched perfusion channel to a second channel, which contained either test compounds dissolved in HL3 or HL3 alone as a vehicle control, for $30 \mathrm{~s}$, after which we switched back to the first HL3 channel for the remainder of the 5 min time course. We purchased all chemicals from Sigma-Aldrich and Fisher Scientific. We performed statistical tests on all live imaging data using Prism 5 (GraphPad) and compared maximum changes in GCaMP3.0 fluorescence or the Epac1-camps inverse FRET ratio between vehicle and test compounds. We used the Mann-Whitney $U$ test for pairwise comparisons of maximum changes, and the Kruskal-Wallis one-way ANOVA with Dunn's post test for multiple comparisons. All plots were generated in Prism 5.

Analysis of locomotor rhythms. We used the Drosophila Activity Monitoring system (Trikinetics) to record the number of beam crosses caused by the fly in $1 \mathrm{~min}$ intervals. Flies were individually placed in glass tubes containing agar-sucrose media. To address the effects of $\mathrm{HB}$ eyelet excitation on free-running locomotor rhythms, we entrained flies expressing the excitatory heat activated cation channel TrpAl in Rh6-expressing photoreceptors to a 12:12 $\mathrm{LD}$ cycle for $7 \mathrm{~d}$ at $20^{\circ} \mathrm{C}$ followed by constant darkness (DD). We delivered a $2 \mathrm{~h}$ heat pulse of $30^{\circ} \mathrm{C}$ at ZT14 during the final night of $\mathrm{LD}$ and continued to record locomotor activity under DD conditions for $10 \mathrm{~d}$ at $20^{\circ} \mathrm{C}$. To prevent signaling from Rh6-expressing photoreceptors of the compound eyes, our experimental flies ( $w$; $h d c^{J K 910} ;$ Rh6-GAL4/UAS-TrpA1) also harbored a null mutation in histidine decarboxylase, which is required for histamine synthesis. The behavior of experimental flies was compared with the behavior of $h d c^{J K 910}$; Rh6-GAL4/+ and hdc ${ }^{J K 10}$; UAS-TrpA1/+ controls.

Raw data were plotted as actograms using ActogramJ (Schmid et al., 2011). To analyze phase shifts, we used the open access program ChronoShop (Dr. Kamil Spoelstra, Netherlands Institute of Ecology, Wageningen, The Netherlands) to determine the center of gravity (COG) (Díez-Noguera, 2013) for each fly for each day of the experiment, as this is the most reliable means to determine the phase of flies lacking input from the compound eyes (Rieger et al., 2003). To determine the COG under entrained conditions, we averaged the COG of the last $2 \mathrm{~d}$ in LD. To determine the phase shift induced by the heat pulse, we calculated the difference between COG on day 1 (COG1) after the heat pulse and COG in entrained conditions. The same was done for day 2 after the heat pulse (COG2). To compensate for differences in the free-running period, we subtracted the shift caused by the free-running period at COG1 and COG2 for each single fly. We calculated the shift caused by the heat pulse as the mean of $\Delta$ COG1 and $\Delta C O G 2$. The free-running period was analyzed using $\chi^{2}$ analysis.

For the determination of the evening peak timing under long days, we entrained flies for $8 \mathrm{~d}$ under LDR 16:8 (a light/dark cycle with $1.5 \mathrm{~h}$ gradual increase or decrease in light intensity after lights-on or before lights-off, respectively). Only the final $5 \mathrm{~d}$ of locomotor activity were used in our analysis. Average activity recorded from day 3-8 was plotted for each fly and the average evening peak time scored as described previously (Schlichting and Helfrich-Förster, 2015). Statistical analysis was performed using ANOVA with a Tukey's multiple-comparisons test for normally distributed data and the Kruskal-Wallis test with a Dunn's multiple-comparisons test for non-normally distributed data. In both cases, $p$ values were adjusted using a Bonferroni correction.

Electrophysiology. We visualized the l-LNvs flies containing the RFPtagged construct $P d f-R F P(y w ; P d f-R F P)$ using a $555 \mathrm{~nm}$ LED light, the use of which prevented the excitation of CRY. Adult flies raised under a $12 \mathrm{~h}: 12 \mathrm{~h}$ LD cycle at $25^{\circ} \mathrm{C}$ were collected $\sim 3-5 \mathrm{~d}$ after eclosion between ZT1 and ZT3, where ZT0 corresponds to lights-on. Flies were decapitated and brains dissected in extracellular saline solution containing the following (in mM): $101 \mathrm{NaCl}, 1 \mathrm{CaCl}_{2}, 4 \mathrm{MgCl}_{2}, 3 \mathrm{KCl}, 5$ glucose, 1.25
$\mathrm{NaH}_{2} \mathrm{PO}_{4}, 20.7 \mathrm{NaHCO}_{3}$, with pH adjusted to 7.2 (Cao and Nitabach, 2008). We transferred dissected brains to saline containing $20 \mathrm{U} / \mathrm{ml} \mathrm{pa-}$ pain with $1 \mathrm{~mm} \mathrm{~L}$-cysteine for $3 \mathrm{~min}$ to digest the ganglion sheath. We then dissected away the photoreceptors, air sacks, and trachea and made a small incision over the position of the l-LNv. Brains were placed ventral side up in the recording chamber, secured using a custom-made anchor (Chen et al., 2015). We perfused brains continuously with aerated $(95 \%$ $\mathrm{O}_{2}, 5 \% \mathrm{CO}_{2}$ ) saline solution. We identified l-LNvs on the basis of their RFP fluorescence, size, and position. We performed a single whole-cell current-clamp recording from one $1-\mathrm{LNv}$ per brain using glass electrodes with $10-20 \mathrm{M} \Omega$ resistance filled with intracellular solution (in $\mathrm{mM}$ ) as follows: $102 \mathrm{~K}$-gluconate, $17 \mathrm{NaCl}, 0.94 \mathrm{EGTA}, 8.5 \mathrm{HEPES}, 0.085 \mathrm{CaCl}_{2}$, $1.7 \mathrm{MgCl}_{2}, \mathrm{pH}$ 7.2. Recordings were made using an Axon MultiClamp 700B amplifier, digitized with an Axon DigiData 1440A (sampling rate: $20 \mathrm{kHz}$; filter: Bessel $10 \mathrm{kHz}$ ), and recorded using pClamp 10 (Molecular Devices). An 1-LNv was included in the analysis if the access resistance was $<70 \mathrm{M} \Omega$ and the leak current in response to a $-40 \mathrm{mV}$ pulse $<-100$ pA. Histamine $(100 \mathrm{~mm})$ was injected in the ipsilateral medulla via a glass pipette (1-3 M $\Omega$ ) and a Picospritzer III (5-10 PSI; Parker Hannifin). All chemicals were purchased from Sigma. The liquid junction potential was calculated as $13 \mathrm{mV}$ and was subtracted from all the membrane voltages.

\section{Results}

The Rhodopsin-6 photoreceptor termini rest in close apposition to the LNvs in the accessory medulla but not in the distal medulla

To express transgenes in the $\mathrm{HB}$ eyelets, we made use of the Rh6-GAL4 driver, as the eyelets express Rhodopsin-6 strongly in the adult (Helfrich-Förster et al., 2002). The R8 photoreceptors of the compound eyes are also targeted by Rh6-GAL4 (Yasuyama and Meinertzhagen, 1999), and we are not aware of a driver that drives strong expression exclusively in the HB eyelets. R8 photoreceptors terminate in the distal medulla of the optic lobes, whereas the eyelets project to the AMe where they terminate near the LNvs and along the ventral elongation of the AMe (HelfrichFörster et al., 2002; Malpel et al., 2002) (Figure 1A-F). If synaptic connections exist between the Rh6-expressing photoreceptors (Rh6-PRs) and the LNvs, split GFP constructs driven independently in these cell types should result in the reconstitution of GFP (Feinberg et al., 2008), whereas a lack of reconstitution would argue against the presence of direct connections between these photoreceptors and the LNvs. Expression of split GFP in Rh6-PRs and LNvs resulted in the reconstitution of GFP specifically in the AMe in 16 of 16 brains imaged. In 13 of these brains, we observed GFP reconstitution along the previously described ventral elongation of the LNv projections (Helfrich-Förster et al., 2002, 2007) (Fig. 1G-L). No GFP reconstitution was detected in other regions of the brain, not even in the distal medulla where R8 termini reside near projections of the l-LNvs. We detected no GFP fluorescence in control parental genotypes containing either the genetic drivers alone or the split GFP elements without drivers (Fig. 1G,I). These results support the hypothesis that the eyelets form direct connections on the LNvs in the AMe and its ventral elongation.

\section{Excitation of Rhodopsin-6 expressing photoreceptors causes $\mathrm{Ca}^{2+}$ and cAMP increases in the small but not the large LNvs and shifts free-running locomotor rhythms}

In the adult fly, the $\mathrm{HB}$ eyelets are immunoreactive to antisera raised against both ChAT and histamine (Pollack and Hofbauer, 1991; Yasuyama and Salvaterra, 1999), suggesting the presence of acetylcholine and histamine in the eyelet nerves. It is not known whether the eyelets use both neurotransmitters in the adult or whether anti-ChAT immunosignals represent a waning pool of ChAT left over from Bolwig's nerves, which is remodeled to be- 

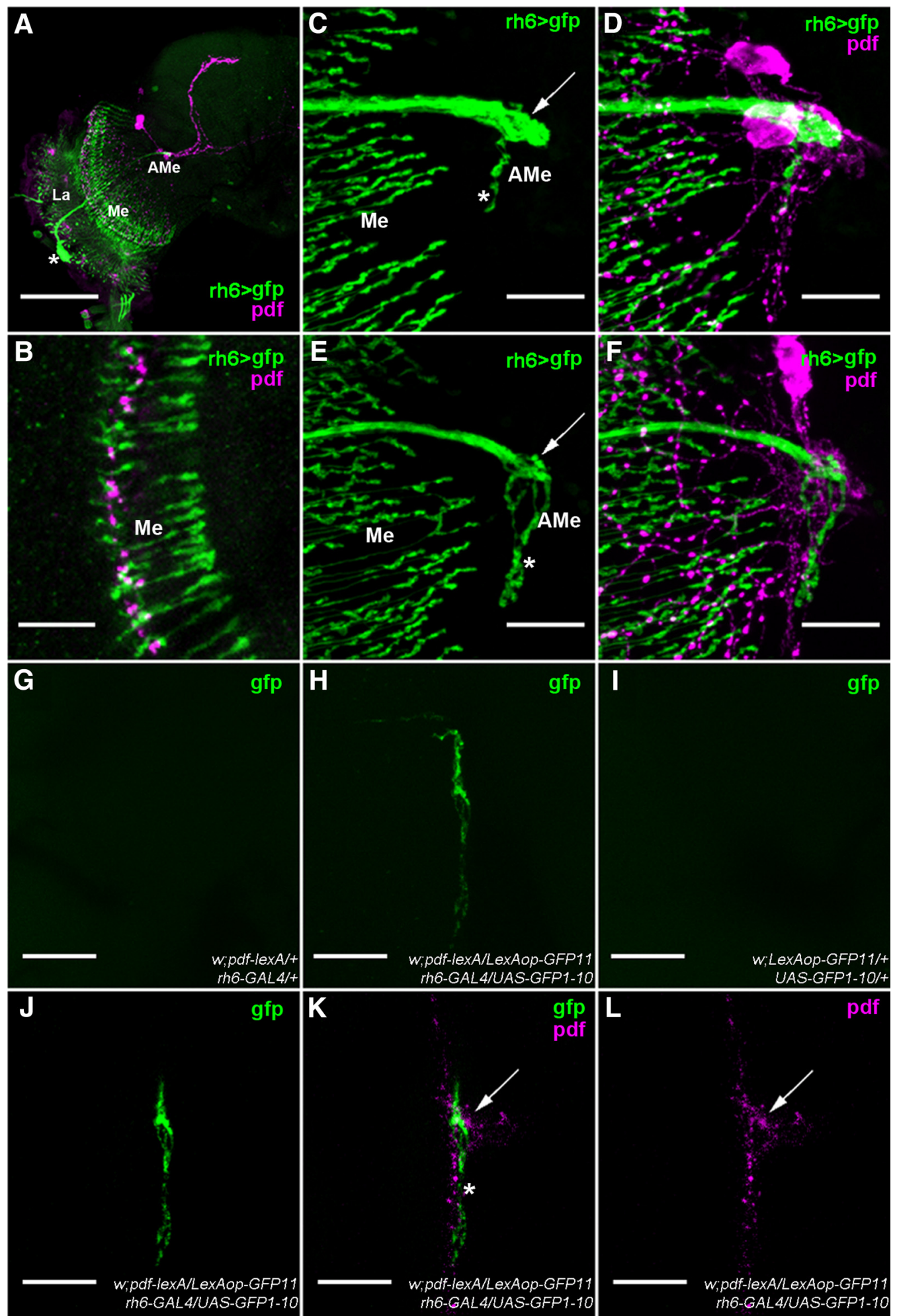

Figure 1. The HB eyelet likely forms synapses directly on the LNv clock neurons within the accessory medulla and along its ventral elongation. $\boldsymbol{A}-\boldsymbol{F}$, The Rh6-GAL4 expression pattern (green) colabeled with anti-PDF (magenta). A, One hemisphere of a UAS-GFP/+;Rh6-GAL4/+ brain coimmunolabeled for GFP and PDF. The axons of the Rh6-positive R8 photoreceptors of the compound eye cross the lamina ( $(\mathrm{La})$ and terminate in the medulla (Me), whereas the axons of the HB eyelet directly innervate the AMe. Scale bar, $100 \mu \mathrm{m} . \boldsymbol{B}$, Axonal terminals of the R8 photoreceptors (green) in the Me reside in close vicinity to the PDF-positive puncta of the I-LNvs (magenta). Scale bar, $15 \mu \mathrm{m}$. $\mathbf{C}-\boldsymbol{F}$, Axons of the HB eyelet terminate in the AMe and overlap with PDF arborizations ( $\boldsymbol{D}, \boldsymbol{F}$, magenta). $(-F$, Micrographs represent $20 \mu \mathrm{m}$ projections of the AMe consisting of 10 optical sections separated by $2 \mu \mathrm{m}$ steps. Rh6-GAL 4 is not expressed in the PDF neurons as no colocalization is visible in the cell bodies of the PDF neurons. Scale bars, $\boldsymbol{C}-\boldsymbol{F}, 15 \mu \mathrm{m} . \boldsymbol{C}, \boldsymbol{D}$, and $\boldsymbol{E}, \boldsymbol{F}$ display two different brains. $\mathbf{G}-\boldsymbol{I}$, Reconstitution of GFP between LNv neurons and the Rh6-positive photoreceptors. G, GFP fluorescence in a Pdf-lexA/+;Rh6-GAL4/+ parental control. H, GFP fluorescence in a Pdf-lexA/lexAop-CD4::spGFP11;Rh6-GAL4/UAS-CD4::sp GFP1-10 brain reveals GFP reconstitution within the AMe and along its ventral elongation. I, GFP fluorescence in a LeXAop-CD4::SpGFP11/+; UAS-CD4.::SpGFP1-10/+ parental control. J-L, Reconstituted GFP (J, $K$, green) in a Pdf-lexA/lexAop-CD4::sp GFP11;Rh6-GAL4/UAS-CD4::spGFP1-10 brain colabeled for PDF ( $K, L$, magenta). Scale bars: $G-L, 25 \mu \mathrm{m}$. $C, E, K, L$, Arrows indicate the central part of the AMe, which is strongly innervated by HB eyelet terminals in all brains. $\boldsymbol{A}$, ${ }^{*} \mathrm{HB}$ eyelet. $\boldsymbol{C}, \boldsymbol{E}, \boldsymbol{K},{ }^{*}$ Ventral elongation of the AMe. 
come the eyelets (Helfrich-Förster et al., 2002; Malpel et al., 2002). It is not known whether either neurotransmitter is used to relay light information from the eyelets to the clock neurons in the AMe, although previous work established that both the l-LNvs and s-LNvs are receptive to acetylcholine (McCarthy et al., 2011; Lelito and Shafer, 2012).

To determine whether an excitatory connection exists between the Rh6-PRs and the LNvs, we rendered the HB eyelet nerves excitable by ATP through the expression of the mammalian purinergic receptor P2X2 (Lima and Miesenböck, 2005) via Rh6-GAL4-mediated expression of UAS-P2X2 (Fig. 2A). When we excited Rh6-PRs, no $\mathrm{Ca}^{2+}$ responses were detected in the 1-LNvs (Fig. 2B). In contrast, the s-LNvs displayed significant $\mathrm{Ca}^{2+}$ increases (Fig. 2C). This excitatory response was not due to nonspecific effects of ATP or to leaky P2X2 expression in the small LNvs, as ATP perfusion in flies lacking the Rh6-GAL4 driver for the directed expression of UAS-P2X2 did not result in s-LNv $\mathrm{Ca}^{2+}$ increases (Fig. 2D). Indeed, in this case, ATP perfusion caused the maximum GCaMP fluorescence changes to be negative in the $\mathrm{s}-\mathrm{LN}_{\mathrm{v}} \mathrm{s}$ (Fig. $2 D$ ). The s-LNvs consistently express lower sensor levels than the l-LNvs; this tendency is accompanied by relatively high sensitivity to movement artifacts, nonspecific effects of bath applied compounds, and bleaching during imaging experiments. Thus, the excitatory response we measure for the s-LNvs in response to Rh6 PR excitation (Fig. 2C) may underestimate the s-LNv response magnitude.

These results suggest that the $\mathrm{HB}$ eyelets provide excitatory drive to the s-LNvs but not the l-LNvs. The presence of antiChAT immunosignals in the eyelet nerve (Yasuyama and Meinertzhagen, 1999) suggests that acetylcholine mediates this excitation. Cholinergic agonists increase cAMP levels in both the l-LNvs and s-LNvs (Lelito and Shafer, 2012). Thus, if the eyelets excite the s-LNvs but not the l-LNvs, excitation of the eyelets should cause cAMP increases in the former but not the latter neuron class. Indeed, excitation of Rh6-PRs produced no significant cAMP changes in the l-LNvs (Fig. 2E) but caused clear increases in cAMP in the s-LNvs (Fig. 2F). Neither the l-LNvs nor s-LNvs displayed cAMP increases in the absence of the GAL4 driver for P2X2 expression (Fig. 2G,H). These results suggest that, among the LNvs, the $\mathrm{HB}$ eyelet specifically excites the s-LNvs.

The acute excitation of LNvs results in phase-dependent advances and delays in free-running locomotor rhythms (Guo et al., 2014). Thus, an excitatory connection between the HB eyelets and the s-LNvs leads to the predication that eyelet excitation will result in phase shifts in the fly's rhythm. We therefore expressed the heat activated cation channel TrpA1 (Hamada et al., 2008) in the R6-PRs by combining UAS-TrpA1 with Rh6-GAL4, thereby rendering the R6-PRs excitable by high temperature $\left(30^{\circ} \mathrm{C}\right)$ pulses. Because a previous study has shown that signals from the compound eyes increase the neuronal firing rate of the l-LNvs (Muraro and Ceriani, 2015), we conducted this experiment in a null $h d c^{K 910}$ mutant, which is unable to synthesize histamine (Burg et al., 1993). In this way, TrpA1-mediated excitation of Rh6-PRs should result in acetylcholine release from the HB eyelets in the absence of histamine release from the R8 photoreceptors in the compound eye or $\mathrm{HB}$ eyelets.

At low temperature $\left(20^{\circ} \mathrm{C}\right)$, the expression of $\operatorname{TrpA} 1$ in the Rh6-PRs of $h d c^{K K 910}$ mutants caused a significant decrease in the free-running period of locomotor rhythms relative to controls (EXP: $23.5 \pm 0.1$; GAL4: $24.3 \pm 0.1$; UAS: $24.3 \pm 0.1$ ). Although dTrpAl channels are gated by temperatures above $\sim 25^{\circ} \mathrm{C}$ (Viswanath et al., 2003) and temperatures $>25^{\circ} \mathrm{C}$ have been ob- served to provoke measurable behavioral changes when $\mathrm{dTrpA} 1$ is overexpressed in neurons of interest (for review, see Bernstein et al., 2012), we cannot exclude the possibility that some dTrpA1mediated currents were present at $20^{\circ} \mathrm{C}$ in $\mathrm{Rh} 6$ photoreceptors overexpressing dTrpA1. Slight depolarization due to such currents could be the cause of the slight period shortening we observed in our experimental flies. We were unable to measure the free-running period of these flies at high temperature due to low survivorship. We excited the Rh6-expressing photoreceptors of experimental flies at ZT 14 with a $2 \mathrm{~h}$ pulse of high temperature, comparing the phase responses of experimental flies to the heat pulse with those of genetic controls that lacked either Rh6-GAL4 or UAS-TrpA1 elements. The heat pulse resulted in a small but significant $(p<0.001)$ phase advance of the free-running behavior in the experimental line ( $88 \pm 14 \mathrm{~min})$ compared with both controls (GAL4: $-3 \pm 15 \mathrm{~min}$; UAS: $4 \pm 13 \mathrm{~min}$ ). A phase advance at this time was surprising, given previous work indicating that the excitation of the large and small LNvs at ZT 15 causes phase delays (Guo et al., 2014). However, recent work has revealed that the specific excitation of the s-LNvs causes only advances in the free-running locomotor rhythm and that LNvinduced delays require the excitation of the l-LNvs (Eck et al., 2016). Thus, HB eyelet excitation modestly but significantly shifted the phase of free-running locomotor rhythms during the early night in a manner consistent with the specific excitation of the s-LNvs.

\section{Histamine inhibits the large LNvs, which govern evening peak phase under long days}

Given the effect of cholinergic input into the CCNN, we wondered whether histamine release from the eyelets can elicit changes in $\mathrm{Ca}^{2+}$ and/or cAMP. Mapping of the inhibitory histamine receptor $\mathrm{HisCl}$ indicated that the large, but not the small, LNvs are receptive to histamine (Hong et al., 2006). We found no evidence that bathapplied histamine caused significant changes in $\mathrm{Ca}^{2+}$ or cAMP in either the l-LNvs or s-LNvs using live imaging methods (data not shown). Histamine acts via inhibitory receptors (Pantazis et al., 2008), and inhibition is often difficult to detect with $\mathrm{Ca}^{2+}$ and cAMP sensors (Lelito and Shafer, 2012), suggesting that histamine either does not inhibit the LNvs or that such inhibition is not detectable with genetically encoded sensors.

Despite our inability to measure histamine responses in the l-LNvs using genetically encoded sensors, the HB eyelet's immunoreactivity to histamine (Pollack and Hofbauer, 1991), the expression of the inhibitory histamine receptor HisCl by the l-LNvs (Hong et al., 2006), and the GRASP results reported above, together suggest that the eyelets act to inhibit the l-LNvs. We therefore performed electrophysiological recordings and asked whether histamine had inhibitory effects on the l-LNvs that were not detectable using our live imaging methods. The l-LNvs are characterized by spontaneous firing (Sheeba et al., 2008). Local application of $100 \mathrm{~mm}$ histamine reliably stopped spontaneous spiking of the l-LNvs $(n=5)$ in a manner dependent on the duration of histamine application (Fig. $2 I, J$ ). In 3 of 5 recordings, histaminergic inhibition of the l-LNvs revealed persistent but much smaller spikes that likely originated from the contralateral l-LNv arbor as previously reported (Cao and Nitabach, 2008). These results suggest that histamine exerts its inhibitory effects locally and briefly, specifically within the hemisphere of histamine application. Although this is consistent with a histaminergic connection between the eyelets and the l-LNvs, we note here that there are other potential sources of histamine in the AMe (Hamasaka and Nässel, 2006). 
A
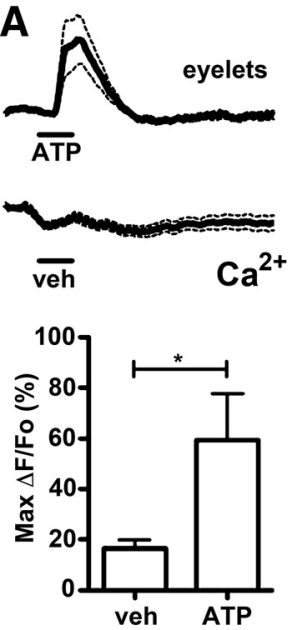

E
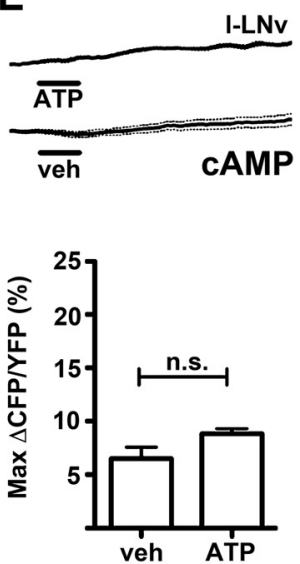

B
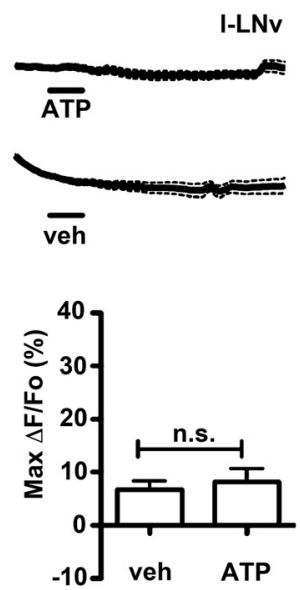

$F$
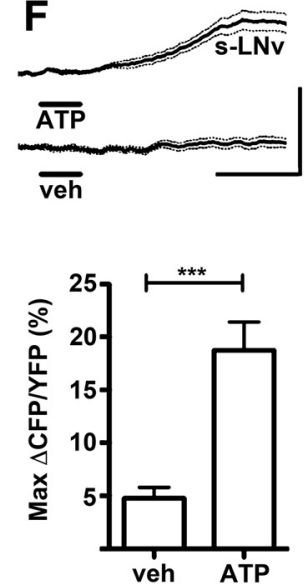

C
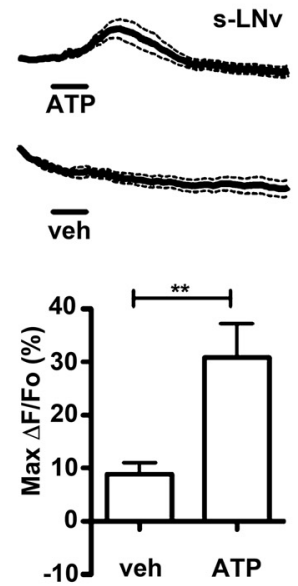

G
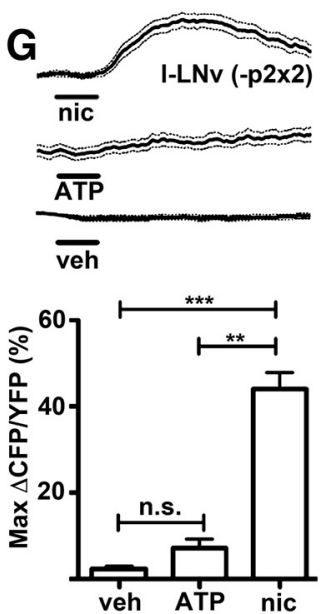

D
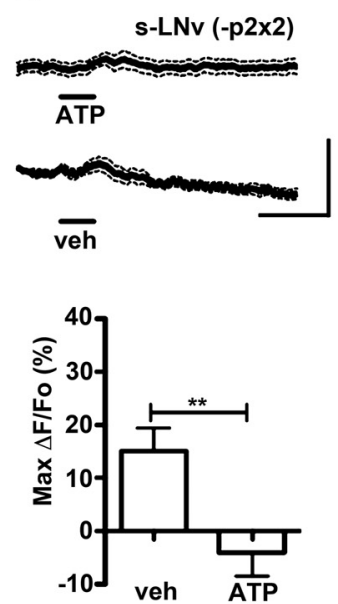

I
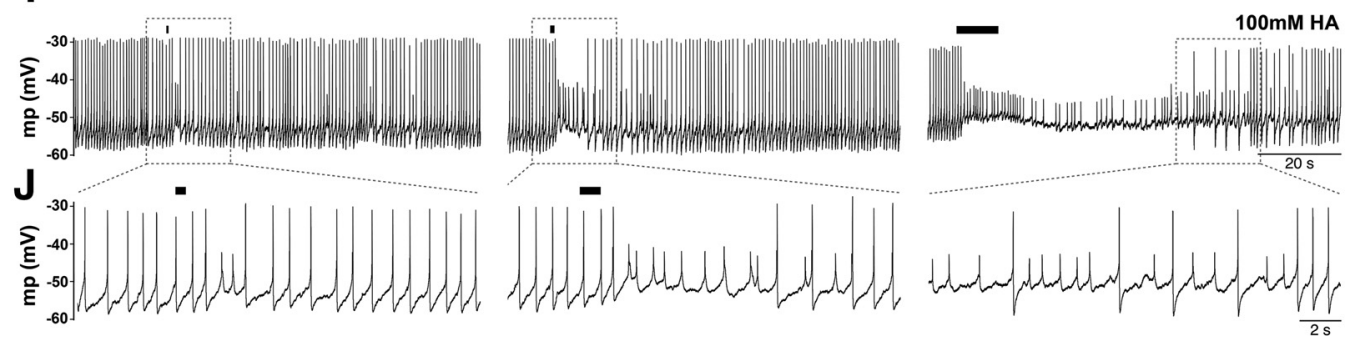

Figure 2. The s-LNvs, but not the I-LNvs, respond to HB eyelet excitation with increases in calcium and CAMP. A, P2X2-mediated excitation of HB eyelets. Top two traces, Average GCaMP3.0 fluorescence plots ( \pm SEM) for HB eyelet nerves coexpressing P2X2 and GCAMP in Rh6-GAL4/UAS-GCaMP3.0;UAS-P2X2/+ brains in response to 30 s perfusion of $1 \mathrm{~mm}$ ATP (top, $N=24$ ) or vehicle (bottom, $N=24$ ). Histogram represents comparison of average maximum GCaMP responses ( \pm SEM) for the same data. ATP caused significant GCaMP fluorescence increases compared with vehicle control. B, Effect of eyelet excitation on I-LNv Ca ${ }^{2+}$ in Pdf-lexA, LeXA-GCaMP3.0/Rh6-GAL4; UAS-P2X2/+ brains in which P2X2 is expressed in the HB eyelet and GCaMP in the LNvs. Data arranged as in $A . N=14$ for ATP and $N=15$ for vehicle. The I-LNvs did not display significant GCaMP fluorescence increases in response to eyelet excitation. $C$, Effect of eyelet excitation on s-LNv Ca ${ }^{2+}$ in Pdf-lexA,LeXAop-GCaMP3.0/Rh6-GAL4;UAS-P2X2/+ brains. Data arranged as in A. $N=20$ for ATP, $N=13$ for vehicle. Excitation of the eyelet resulted in significant GCaMP fluorescence increases relative to vehicle controls. $\boldsymbol{D}$, In brains lacking a driver for UAS-P2X2 expression in the eyelets (Pdf-lexA,LexAop-GCaMP3.0/+;UAS-P2X2/+). ATP failed to result in an increase in s-LNv GCaMP fluorescence relative to vehicle controls. Data arranged as for $\boldsymbol{A} . N=13$ for ATP, $N=13$ for vehicle. Calibration: $\boldsymbol{A}-\boldsymbol{D}, 1 \mathrm{~min}$ ( $X$-axis) and a $50 \%$ change in GCaMP3.0 fluorescence over baseline (y-axis). E, Effect of eyelet excitation on I-LNv cAMP in Pdf-lexA,LeXAop-Epac1-camps/Rh6-GAL4;UAS-P2X2 brains. The two traces represent average inverse Epac1-camps FRET (CFP/YFP) plots for I-LNvs in response to $1 \mathrm{~mm} \mathrm{ATP} \mathrm{(top,} N=17$ ) and vehicle (bottom, $N=17$ ). Histogram represents comparison of average maximum Epac1 camps responses ( \pm SEM) for the same data. ATP perfusion failed to produce significant inverse FRET increases in I-LNvs relative to vehicle controls. F, Excitation of the eyelet causes CAMP increases in the s-LNvs of Pdf-lexA,LexAop-Epac1-camps/Rh6-GAL4; UAS-P2X2. Data arranged as for E. ATP produced significant inverse FRET increases in the s-LNvs relative to vehicle controls. $N=15$ for ATP, $N=14$ for vehicle. G, Average inverse Epac1-camps FRET (CFP/YFP) plots for I-LNvs in Pdf-lexA,LeXAop-Epac1-camps/+;UAS-P2X2/+ brains that express Epac1-camps in the LNvs but fail to drive P2X2 in the eyelet. The I-LNvs did not display inverse FRET increases in response to ATP (middle, $N=14$ ) relative to vehicle controls (bottom, $N=14$ ). The l-LNvs did display inverse FRET increases to $10^{-4} \mathrm{M} \mathrm{nicotine} \mathrm{(top} \mathrm{plot,} N=12$ ). Histogram represents comparison of maximum Epac1-camps responses for the same data. ATP perfusion failed to produce significant increases in CYP/YPF ratio in the I-LNvs relative to vehicle controls, whereas nicotine $\left(10^{-4} \mathrm{M}\right)$ produced significant CAMP increases. $\boldsymbol{H}$, Average inverse Epac1-camps FRET (CFP/YFP) plots for s-LNvs in Pdf-lexA,LeXAop-Epac1-camps/+;UAS-P2X2/+ brains. Data organized as for G. ATP perfusion $(N=19)$ caused small but significant increases in CYP/YPF ratio relative to vehicle controls $(N=19)$ in the s-LNvs, whereas nicotine $\left(10^{-4} \mathrm{M}, N=15\right)$ produced large and significant inverse FRET increases. Calibration: $\boldsymbol{E}-\boldsymbol{H}, 1 \mathrm{~min}$ ( $x$-axis) and a $25 \%$ changes in (FP/YFP ratio (y-axis). Bars under averaged plots represent 30 s of ATP perfusion switched from a constant saline flow. n.s., Not significant $(p \geq 0.05) .{ }^{*} p<0.05 .{ }^{* *} p<0.01 .{ }^{* *} p<0.001$. I, Response of a representative I-LNv to local pressure injection of 100 mm histamine (Figure legend continues.) 

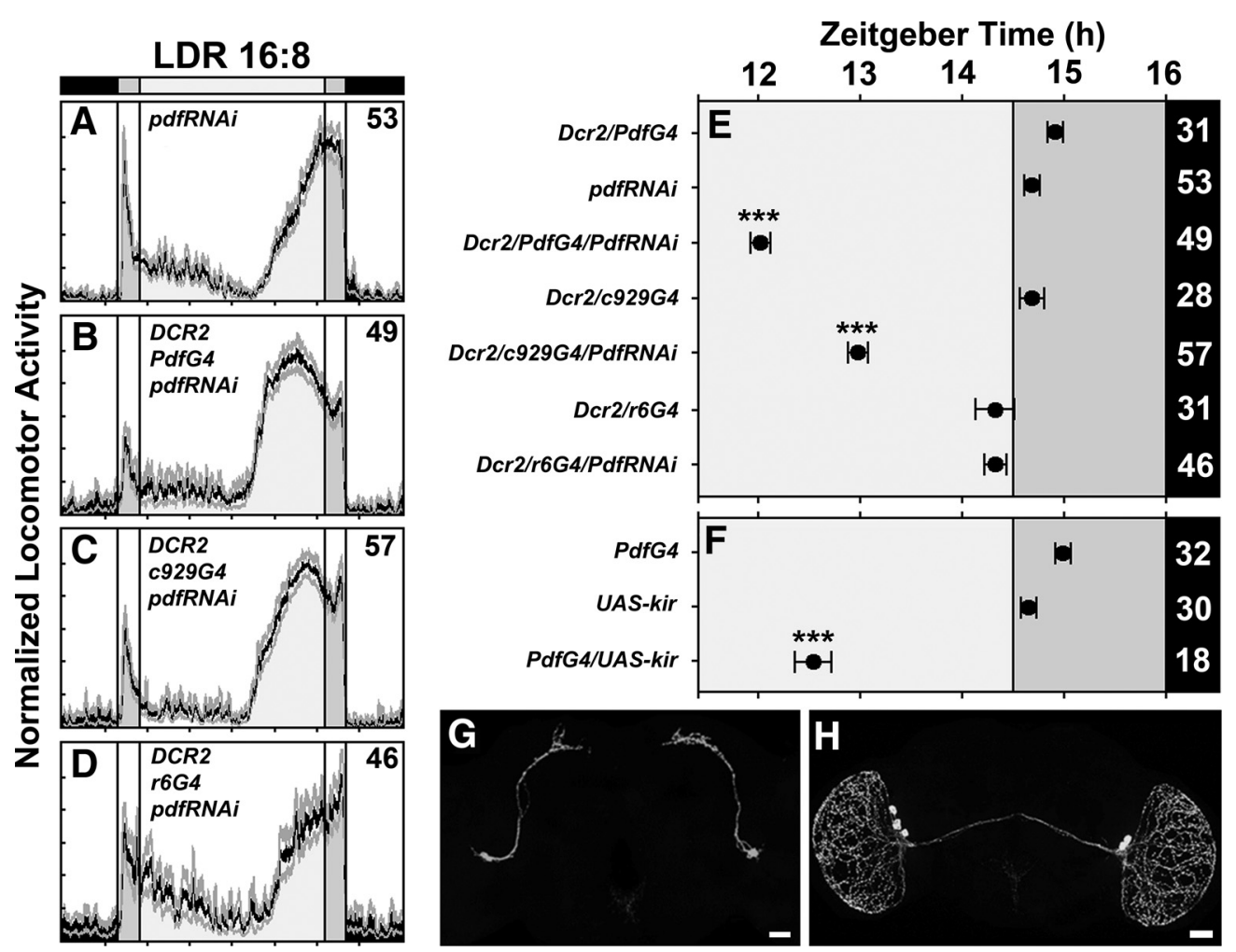

Figure 3. PDF is required specifically in the I-LNvs for normally phased evening peaks under long day conditions. $A$, The averaged activity profile of a UAS-PdfRNAi control flies under LDR 16:8. The morning and evening peaks are aligned with dawn and dusk (light gray regions of the LD cycle above the plot). $B$, Activity of flies in which PDF has been knocked down in all LNvs through the coexpression of UAS-PdfRNAi and UAS-Dicer2 (Dcr2) under the control of Pdf-GAL4. C, Activity of flies in which PDF has been knocked down only in the I-LNvs using the c929-GAL4 driver (D). Activity of flies in which PDF has been knocked down only in thes-LNvs using the r6-GAL4 driver. $\boldsymbol{E}$, The average evening peak phase of flies in which Pdf expression has been knocked down in different subsets of neurons. Knockdown of Pdf either in all LNvs or specifically in the I-LNvs, using Pdf-GAL4 and C929-GAL4, respectively, resulted in a significantly advanced evening peak of activity compared with Dicer2 overexpression and UAS-PdfRNAi controls. Knockdown of Pdfspecifically in the s-LNvs using R6-GAL4 failed to produce an advanced evening peak relative to controls. ANOVA followed by a post hoc test revealed no significant differences in phase between Dcr2;PdfG4, PdfRNAi, Dcr2/c929G4, Dcr2/r6G4, and Dcr2/r6G4/PdfRNAi flies ( $p>0.1199)$. However, Dcr2/PdfG4/PdfRNAi and $D c r 2 / c 92964 / P d f R N A i$ flies had a significant earlier evening peak than all others $(p<0.001)$. In addition, the evening peak phase between these two lines was significantly different $(p<0.001)$. $\boldsymbol{F}$, The average evening peak phase under LDR 16:8 of flies in which the LNvs have been electrically silenced (bottom row:Pdf-GAL4/UAS-Kir2.1). As expected, these flies display an abnormally early evening peak of activity compared with Pdf-GAL4 (top row) and UAS-Kir2.1 (middle row) controls (Kruskal-Wallis test, $p<0.001$ ). A-F, Numbers on the right side of the panels indicate sample size. G, PDF expression in a representative UAS-Dicer2/c929-GAL4/UAS-PdfRNAi brain. Only s-LNv PDF is visible. H, PDF expression in a UAS-Dicer2/R6-GAL4/UAS-PdfRNAi brain. Only I-LNv PDF is visible. Scale bars: $\mathbf{G}, \boldsymbol{H}, 20 \mu \mathrm{m}$. In these experiments, the GAL4 and UAS elements were always present as single copies. The GAL4 elements and UAS-RNAi elements were autosomal while the UAS-Dcr2 element was inserted into the $X$ chromosome. Thus, flies were heterozygous for GAL4 and UAS-RNAi and hemizygous for the UAS-Dicer2 element. ${ }^{* * *} p<0.001$.

Given the modulatory effect of histamine on the 1-LNvs, we wondered whether changing the properties of these neurons might affect the behavior of flies. Flies lacking PDF peptide or LNvs display abnormally advanced peaks of evening activity (Renn et al., 1999). Previous work suggested that PDF from either the l-LNvs or the s-LNvs is sufficient for the normal timing of the evening peak under equinox (Shafer and Taghert, 2009), suggesting that PDF released from the l-LNvs, though not required for normal evening peak phase, nevertheless contributes to evening peak timing. We asked whether PDF released from the l-LNvs might be required for normally timed evening peak phase under long day conditions. As previously shown for equinox, the knockdown of PDF from all LNvs resulted in a significant advance in the evening peak of activity (Fig. $3 A, B, E$ ) in a manner reminiscent of the loss of PDF neuron function (Fig. $3 F$ ). In

$\leftarrow$

(Figure legend continued.) $\quad$ (HA) into the ipsilateral Me. Black bars represent stimulus length $(0.5 \mathrm{~s}, 1 \mathrm{~s}, 10 \mathrm{~s})$. Duration-dependent inhibition of ipsilateral spiking activity (large spikes) was apparent, although contralateral activity (small spikes) was not affected.J, Enlargements of the boxed regions in I showing the inhibition after drug application. Spiking stops abruptly but comes back gradually before returning to previous levels. mp, Membrane potential. contrast to previous experiments under LD 12:12, knockdown of PDF specifically in the l-LNvs resulted in a significant advance in the evening peak under long days (Fig. $3 C, E, G$ ), whereas the knockdown of PDF specifically in the s-LNvs had no obvious effects on the evening peak compared with controls under long day conditions (Fig. 3D,E,H). The knockdown of PDF in both the l-LNvs and s-LNvs produced a larger effect on the evening phase than the knockdown in the l-LNvs alone (Fig. $3 B, C, E$ ). This difference is likely a reflection of differing levels of GAL4mediated RNAi expression by Pdf-GAL4 and c929-GAL4.

Excitation of the 1-LNvs produces pigment-dispersing factor receptor-dependent cAMP increases in the s-LNvs

The finding that PDF is required specifically in the l-LNvs for the normal phasing of the evening peak of activity under long days suggested that these neurons, previously characterized as wakepromoting neurons with limited control over circadian timekeeping (Parisky et al., 2008; Shang et al., 2008; Sheeba et al., 2008; Chung et al., 2009), might modulate the evening cells of the CCNN, either directly or indirectly through actions on the $s$-LNvs. Anatomical evidence suggests that the large LNvs might modulate the critical s-LNv pacemakers: projections from the 

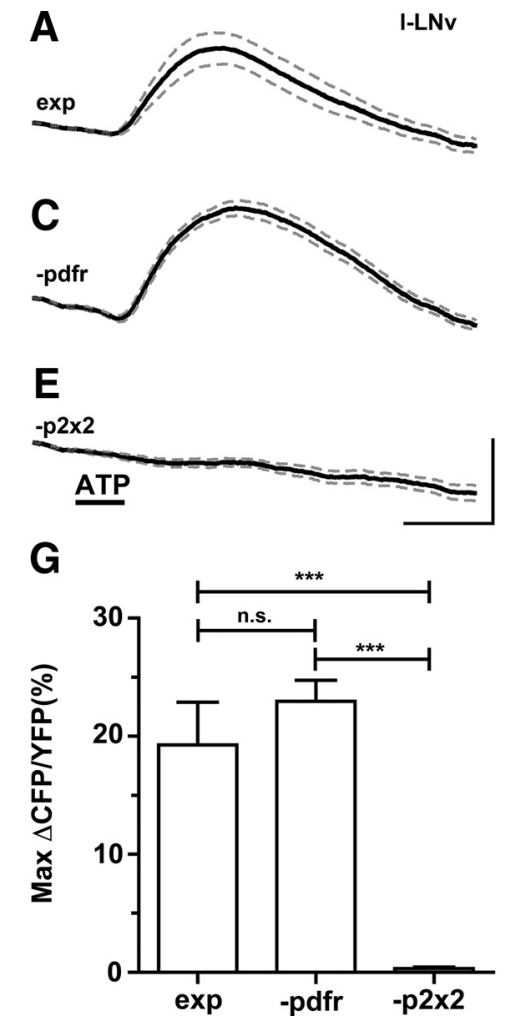

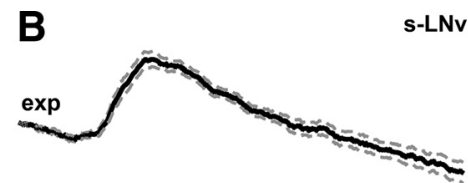

D

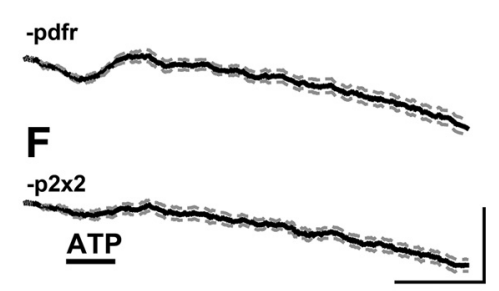

H

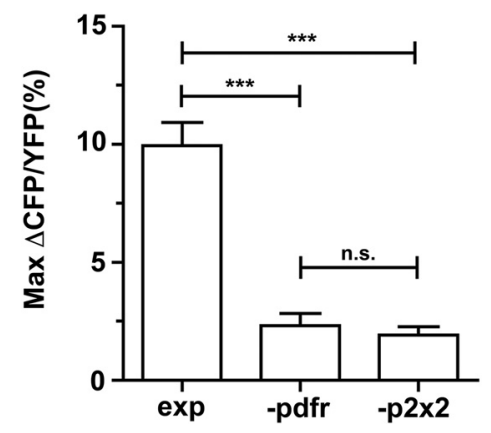

Figure 4. The I-LNvs modulate cAMP levels in the $s$-LNvs. $A$, Averaged Epac1-camps inverse FRET plot ( \pm SEM) of I-LNvs imaged in C929-Gal4/Pdf-LexA,LeXAop-Epac1-camps;UAS-P2X2/+ brains before, during, and after 30 s perfusion of $1 \mathrm{~mm} \mathrm{ATP} \mathrm{(indicated}$ on the bottom plot in each column). ATP/P2X2-mediated excitation caused clear inverse FRET increases. $B$, Averaged Epac1-camps inverse FRET plot ( \pm SEM) of s-LNvs from the same brains as in $\boldsymbol{A}$. Excitation of the 929 network produced inverse FRET increases in the s-LNvs. C, Averaged Epac1-camps inverse FRET plot ( \pm SEM) of large LNvs imaged in a Pdfr mutant background using han ${ }^{5304} ;$;929-GAL4/Pdf-LeXA,LeXAop-Epac1-camps;UAS-P2X2/+ brains. 929 network excitation caused clear inverse FRET increases in these neurons. D, Averaged Epac1-camps plot ( \pm SEM) of s-LNvs from the same brains as in $\boldsymbol{C}$. Excitation of the 929 network failed to produce inverse FRET increases in the $s$-LNvs in the absence of PdfR function. $\boldsymbol{E}$, Averaged Epac1-camps inverse FRET plot ( \pm SEM) of large LNvs imaged in Pdf-LexA,LeXAop-Epac1-camps/+;UAS-P2X2/+ brains. ATP failed to produce inverse FRET increases in the absence of the GAL4 driver. Calibration: $60 s$ ( $x$-axis) and a 20\% change in inverse FRET ( $y$-axis) (also apply to $\boldsymbol{A}, \boldsymbol{C})$. $\boldsymbol{F}$, Averaged Epac1-camps inverse FRET plot ( \pm SEM) of s-LNvs from the same brains as in $\boldsymbol{E}$. ATP caused no obvious inverse FRET increases. Calibration: $\boldsymbol{F}, 60 \mathrm{~s}$ ( $x$-axis) and a 10\% change in inverse FRET (y-axis) (also apply to $\boldsymbol{B}, \boldsymbol{D}$ ). $\boldsymbol{G}$, Comparison of maximum Epac1-camps responses for the I-LNv data shown in $\boldsymbol{A}, \boldsymbol{C}, \boldsymbol{E}$. ATP $(1 \mathrm{~mm})$ perfusion caused significant inverse FRET increases in both the experimental ("exp" (929-GAL4/Pdf-LeXA,LexAop-Epac1-camps;UAS-P2X2/+) and PdfR mutant ("-pdfr" han ${ }^{5304} ;$;929-GAL4/Pdf-LeXA,LeXAop-Epac1-camps;UAS-P2X2/+) conditions, relative to the negative control lacking the GAL4 driver for P2X2 expression ("-p2x2" Pdf-LeXA,LexAop-Epac1-camps/+;UAS-P2X2/+). $\boldsymbol{H}$, Comparison of maximum Epac1-camps responses for the s-LNv data shown in $\boldsymbol{B}, \boldsymbol{D}, \boldsymbol{F}$. ATP (1 mM) perfusion caused significant inverse FRET increases in experimental (exp) flies relative to both PdfR mutants (-pdfr) and $-p 2 \times 2$ controls. Genotypes were identical to those in $\mathbf{G} . \mathbf{G}, \boldsymbol{H}_{\text {, }}^{* * *} p<0.001$. n.s., No significant difference $(p \geq 0.05)$.

1-LNvs reside in close apposition to s-LNv dendrites (HelfrichFörster et al., 2007) and the s-LNvs are receptive to PDF (Shafer et al., 2008; Im and Taghert, 2010). The excitation of the c929GAL4-positive neurons, which include the l-LNvs, does not produce excitatory $\mathrm{Ca}^{2+}$ responses in the s-LNvs (Yao et al., 2012). Given that the PDF receptor signals predominantly through increases in cAMP (Hyun et al., 2005; Mertens et al., 2005; Shafer et al., 2008), we asked whether the s-LNvs responded to the excitation of the l-LNvs with cAMP increases by expressing the P2X2 receptor in the c929-GAL4-positive neurons of the brain while simultaneously expressing the cAMP sensor Epac1-camps in the 1-LNvs and s-LNvs. As expected, ATP-mediated excitation of the c929-positive network in c929-GAL4/Pdf-LexA, LexAop-Epac1camps;UAS-P2X2/+ brains resulted in excitatory cAMP responses in the l-LNvs (Fig. 4A,G). Excitation of the l-LNvs was accompanied by cAMP increases in the s-LNvs (Fig. $4 B, H$ ), suggesting the presence of a modulatory connection between c929-positive neurons and the s-LNvs.

Although $c 929-G A L 4$ is expressed in the 1-LNvs but not the s-LNvs, it is also expressed in many other peptidergic neurons of the CNS (Hewes et al., 2003). To determine whether the l-LNvs were responsible for the cAMP response in the s-LNvs, we repeated the c929-GAL4 excitation experiment in a loss-of-function PdfR mutant $\left(h^{5} n^{5304}\right)$ background. Perfusion of ATP caused excitatory cAMP responses in the lLNvs of han ${ }^{5304}$;c929-GAL4/Pdf-LexA, LexAop-Epac-1camps;UAS-P2X2/+ brains (Fig. 4C, $G$ ) but failed to produce significant cAMP increases in the s-LNvs (Fig. $4 D, H$ ), suggesting that PDF released from the 1LNvs was responsible for the effects of $c 929$ network excitation on the s-LNvs. Flies expressing cAMP sensor in the LNvs that contained the UAS-P2X2 element but lacked the c929-GAL4 driver displayed no responses to bath applied ATP in either the l-LNvs or the s-LNvs (Fig. $4 E-H$ ), indicating that the excitatory responses in the $1-$ LNvs in the previous experiments were indeed due to the specific expression of $\mathrm{P} 2 \mathrm{X} 2$ in the $c 929$ network. These results reveal a modulatory connection from the 1LNvs to the s-LNvs and indicate that PDF released from the former neurons accounts, at least in part, for the activation of PDF receptor in the s-LNvs.

Expression of PdfR in four evening cells is sufficient for the normal phase of evening activity under long days Given the presence of a modulatory connection between the large and small LNvs, the l-LNvs might influence the phase of the evening peak by modulating the clock within the s-LNvs. (We note here that PDF is not the only transmitter produced by the s-LNvs and that these neurons are anatomically well suited to modulate most components of the CCNN.) Like $p d f^{01}$ mutants and flies with reduced PDF in the l-LNvs, loss-offunction $p d f r$ mutants display an abnormally advanced evening peak (Hyun et al., 2005). This was also clear in $p d f r$ mutants observed under LDR 16:8 (Fig. 5A,F). If the l-LNvs adjust the evening peak of activity through the modulation of the s-LNvs, then we would predict that $p d f r$ would be required in the s-LNvs for normal evening peak phase under LDR 16:8. Rescue of $p d f r$ expression in the LNvs (Pdf-GAL4) or specifically in the s-LNvs (r6-GAL4) of $\mathrm{han}^{5304}$ mutants failed to produce a normally phased evening activity peak relative to han ${ }^{5304}$;UAS-pdfr controls (Fig. $5 B, F$ ). This result is not surprising given that $p d f r$ is likely required in evening cells for the reception of PDF from either class of LNv. To challenge this idea, we rescued $p d f r$ expression within the evening cells via the R78G02-GAL4 driver, which is expressed in the fifth s-LNv and the three cry-expressing LNds, in addition to $\sim 15$ neurons outside of 

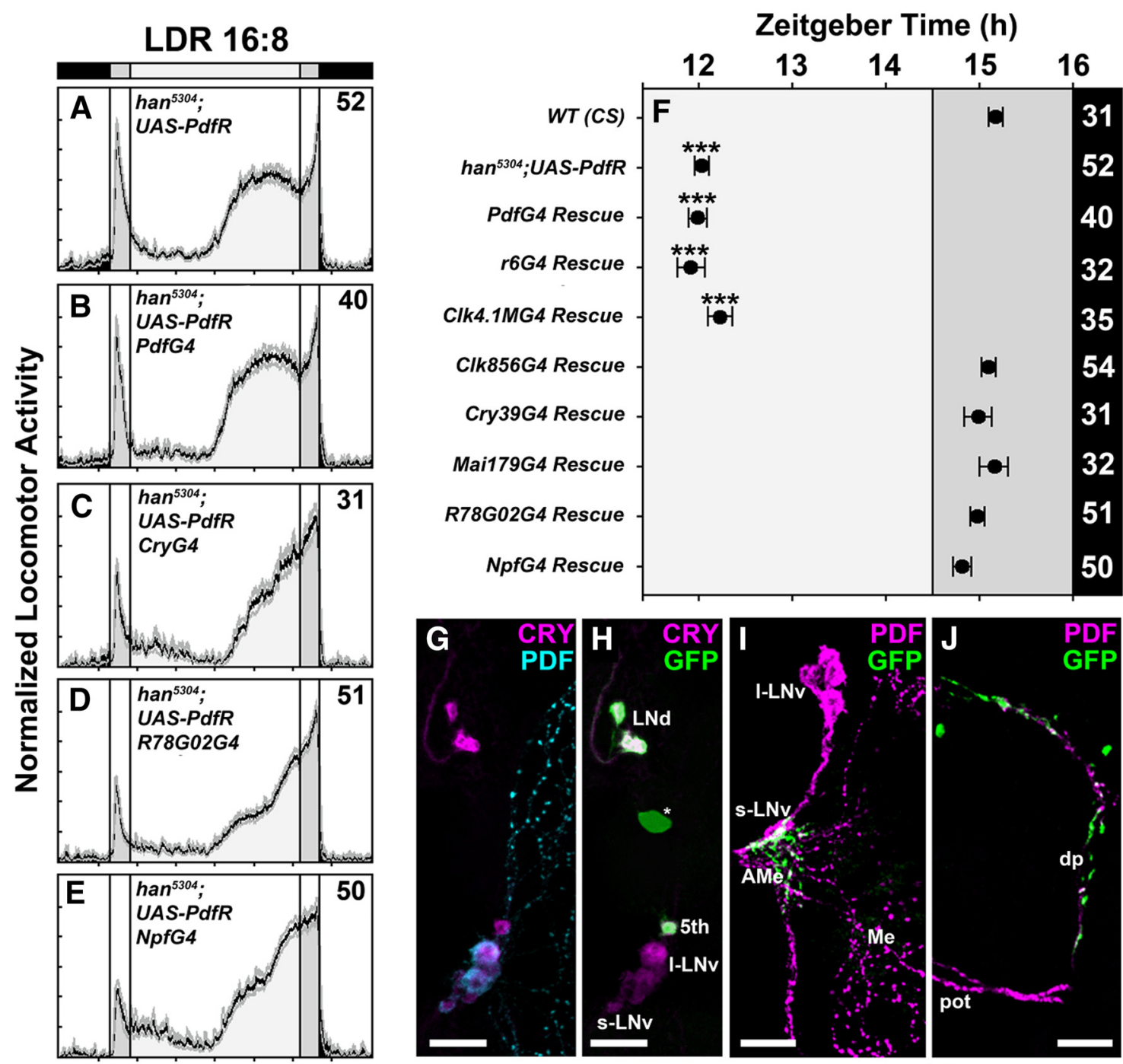

Figure 5. PdfR expression in a small group of evening cells is sufficient for normal evening peak phase under long days. $A$, The averaged activity profile of han ${ }^{5304} ; U A S-P d f r$ control flies under LDR 16:8. The evening peak is advanced compared with wild-type controls (compare withFig. 4C). B, The averaged activity profile of han ${ }^{5304}$;Pdf-GAL4/UAS-Pdfr flies. Pdfr expression in the I-LNvs and $s$-LNvs is not sufficient to rescue normal evening peak phase. C, The averaged activity profile of han $^{5304}$; Cry-GAL4/UAS-Pdff flies. The rescue of Pdfr expression in the cry- expressing clock neurons is

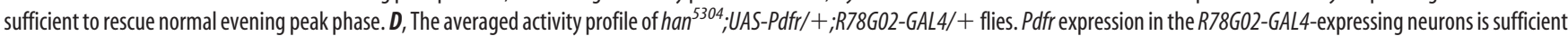
to rescue normal evening peak phase. $\boldsymbol{E}$, The averaged activity profile of $h a n^{5304}$;npf-GAL4/UAS-Pdfr flies. Pdfr expression in the NPF expressing clock neurons is sufficient to rescue normal evening peak phase. $\boldsymbol{F}$, A comparison of evening peak phases between wild-type (WT) flies, flies lacking functional Pdfr (PdfR-/UAS-PdfR), and flies in which Pdfr expression has been rescued in various subsets of clockneurons using various GAL4 (G4) drivers. Only rescues that included the fifth s-LNv-and CRY-positive LNds (bottom five genotypes) resulted in rescue of evening peak under LDR 16:8. ANOVA followed by a post hoc test revealed highly significant differences in evening peak phase between the fly lines marked by asterisks and the ones without asterisks $(p<0.001)$. The lines with asterisks were not significantly different from each other $(p=1.000)$. Neither were the unmarked lines significantly different from each other ( $p>0.838) . \mathbf{G}, \mathrm{PDF}$ (cyan) and (RY (magenta) expression in a single hemisphere of a UAS-StingerGFP/+;R78G02-GAL4/+ brain. Only the region bordering the central brain and Me is shown. $\boldsymbol{H}$, The same brain region as in $\boldsymbol{G}$ coimmunolabeled for GFP (green) and CRY (magenta). R78G02-GAL4 drives GFP expression in the CRY-positive LNds and the fifth s-LNv. Another, nonclock neuron-expressing GFP (asterisk) is also present in this brain region. $I$, A GRASP brain preparation in which GFP (green) has been reconstituted between LNvs (magenta) and R78G02-GAL4 expressing neurons. Reconstituted GFP is visible in the AMe near the s-LNvs. J, A GRASP brain preparation as in I. Reconstituted GFP is visible along dorsal projection (dp) of the s-LNvs. pot, Posterior optic tract of the I-LNvs. Scale bars, G-I, $20 \mu \mathrm{m}$. ${ }^{* * *} p<0.001$.

the clock network (Fig. 5G,H). In these flies, the evening activity peak displayed a delayed phase compared with $p d f^{-}$controls (Fig. $5 D, F$ ). To confirm this finding, we rescued $p d f r$ expression with several other GAL4 drivers. Expression of $p d f r$ with all driver lines, including these neurons (Clk856-GAL4, cry39-GAL4, and Mai179-GAL4), rescued the phase of the evening peak (Fig. 5C,F). Conversely, the expression of $p d f r$ using the Clk4.1M-GAL4, a driver expressed only in the DN1p class of clock neurons, failed to rescue the phenotype (Fig. $5 F$ ).

Together, our $p d f r$ rescue results suggest that $p d f r$ expression within the fifth s-LNv and a subset of the LNds is sufficient for normal evening peak phase under long days. When used in con- junction with $P d f-L e x A$, GRASP experiments with R78G02-GAL4 revealed the reconstitution of GFP within the AMe and along the dorsal projection of the s-LNvs (Fig. $5 I, J$ ), consistent with synaptic connections between the LNvs and the R78G02-GAL4 positive network. Neuropeptide F (NPF) is expressed by the fifth s-LNv and is also expressed in one R78G02-GAL4 positive LNd in each hemisphere. These neurons also express ITP (Johard et al., 2009). Rescue of $p d f r$ expression using $n p f-G A L 4$ almost completely rescued the evening peak phase (Fig. $5 E, F$ ) revealing that pdfr expression in the NPF/ITP-positive LNd and fifth s-LNv is largely sufficient for the normal phasing of the evening peak under long days. This indicates that the $p d f r$-dependent modulatory 


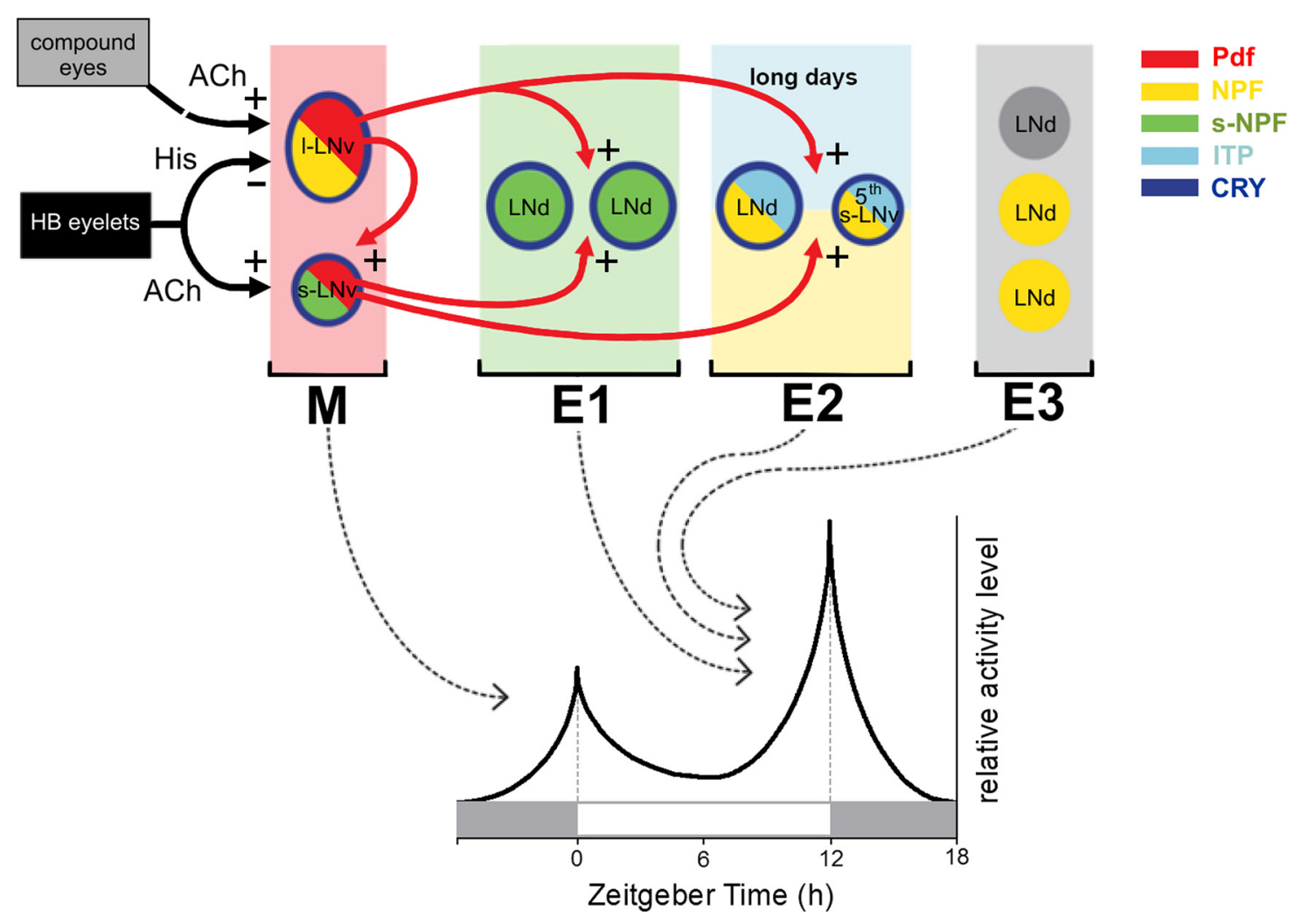

Figure 6. Network model of light input in the circadian clock neuron network that governs bimodal locomotor rhythms. The I-LNvs receive excitatory cholinergic from the compound eyes (ACh) and inhibitory histaminergic (His) input from the HB eyelets, whereas the s-LNvs receive excitatory ACh input from the HB eyelets. Thus, HB eyelet activity antagonizes compound eye- and CRY-mediated light excitation in the I-LNvs while synergizing CRY-mediated excitation in the s-LNvs. Although we separate ACh and His terminals for the eyelet in the model, we do not mean to suggest that these two transmitters are differentially trafficked to different presynaptic termini. Rather, the differential effects of eyelet excitation are most likely due to the differential expression of receptors on the I-LNvs and s-LNvs. The I-LNvs modulate (red arrows) the s-LNvs and the E1 and E2 evening neurons via PDF. Such modulatory signaling likely sets the phase of the molecular clocks in target neurons, although it results in a modest excitation of targets as well (see Discussion). We hypothesize that the effects of the HB eyelets wane over the course of the day. Consequently, the excitatory influence of the compound eyes on the I-LNvs prevails under long days. The neuropeptides expressed by the various classes of clock neurons are indicated by color as is the pattern of CRY expression (dark blue outline). For the exception of the I-LNvs, PdfR is expressed wherever CRY is expressed. M, Morning neurons (s-LNv); E3, evening neurons that are not receptive to PDF.

connection between the l-LNvs and the s-LNvs is not required for the adjustment of the evening peak by the l-LNvs under long day conditions.

\section{Discussion}

The experiments described above lead to two unexpected findings regarding the network properties of circadian entrainment in Drosophila. First, the l-LNvs govern the phase of evening peak of activity through PdfR-dependent effects on evening cells that bypass the s-LNvs. Although previous work has implicated the l-LNvs in the control of evening peak phase (Cusumano et al., 2009; Potdar and Sheeba, 2012), our results are the first to provide evidence that there is a direct connection between the l-LNvs and evening cells within the AMe and that this connection mediates the photoperiodic adjustment of sleep and activity in the fly. Second, the HB eyelets light input pathways, long implicated in circadian entrainment, have opposing effects on the l-LNvs and s-LNvs, inhibiting the former and exciting the latter. These results reveal not only a differential effect of a light input pathway on specific nodes of the CCNN but also establish that light from extraretinal photoreceptors can have synergistic or antagonistic effects on CRY- and compound eye-mediated light responses, depending on the clock neuron target in question.

Both the l-LNvs and s-LNvs express the blue light circadian photoreceptor CRY, the expression of which renders neurons directly excitable by light entering the brain through the cuticle (Fogle et al., 2011). How such CRY-mediated light input interacts with input from external photoreceptors is not well understood, although it is known that each system alone is sufficient for the entrainment of locomotor rhythms (Helfrich-Förster et al., 2001). Genetic evidence suggests that the HB eyelets have relatively weak effects on circadian entrainment: flies with functional eyelets that lack compound eyes, ocelli, and CRY entrain relatively poorly to LD cycles relative to flies with functional eyes or CRY (Rieger et al., 2003). The small phase responses of locomotor rhythms to HB eyelet excitation further supports a relatively weak effect of the eyelet on free-running locomotor rhythms.

The LNvs are critical nodes in the CCNN and are closely associated with input pathways linking the central brain to external photoreceptors (Helfrich-Förster et al., 2007). Work on the LNvs has provided evidence for a division of labor among the l-LNvs and s-LNvs: the l-LNvs are wake-promoting neurons that acutely govern arousal and sleep independently of the s-LNvs (Parisky et al., 2008; Shang et al., 2008; Sheeba et al., 2008; Chung et al., 2009), whereas the s-LNvs act as key coordinators of the CCNN to support robust circadian timekeeping (Helfrich-Förster, 1998; Grima et al., 2004; Shafer and Taghert, 2009; Sheeba et al., 2010). Anatomical and genetic evidence has long supported the notion that the dorsal projections of the s-LNvs represent the key connection between the LNvs and the remaining components of the CCNN (Helfrich-Förster, 1998; Grima et al., 2004; Fernández et 
al., 2008; Sivachenko et al., 2013; Petsakou et al., 2015). However, a smaller body of work has suggested that the l-LNvs also contribute to the entrainment of sleep/activity rhythms under LD cycles (Shang et al., 2008; Shafer and Taghert, 2009; Sheeba et al., 2010). Our Pdf knockdown and PdfR rescue experiments under long day conditions indicate that, as the day grows longer, the 1-LNvs play a greater role in the timing of the evening peak (Fig. 3; compared with Shafer and Taghert, 2009). Moreover, the effects of PDF released from the l-LNvs are mediated not by the PDF receptive s-LNvs but rather by the fifth s-LNvs and a subset of the LNds (Fig. 5), the NPF and ITP coexpressing LNds in particular (with some influence of the other PDF-receptor positive LNds). These same neurons were recently identified as evening cells that are physiologically responsive to PDF but relatively weakly coupled to LNv clocks under conditions of constant darkness (Yao and Shafer, 2014). Our results suggest that the l-LNvs differentially modulate the NPF/ITP-positive evening oscillators as a function of day length, producing stronger PDF-dependent delays under long day conditions through increased release of PDF from the l-LNvs, thereby delaying the evening activity peak. Thus, the l-LNvs mediate their effects on the evening peak of activity through their action on the NPF/ITP-positive subset of evening oscillators. The proposed PDF release from the l-LNvs under long days requires their activation via CRY and/or the compound eyes via ACh release from lamina L2 interneurons (Muraro and Ceriani, 2015). We hypothesize that the inhibitory influence of the $\mathrm{HB}$ eyelets ceases under long days allowing the compound eyes and CRY to maximally excite the l-LNvs. Indeed, previous work has established that the compound eyes are especially important for adapting fly evening activity to long days (Rieger et al., 2003). Furthermore, several studies have suggested that the compound eyes signal to the l-LNvs leading to enhanced PDF release and a slowing-down of the evening oscillators (Rieger et al., 2006; Wülbeck et al., 2009; Yoshii et al., 2009; Helfrich-Förster, 2014). A recent paper measuring $\mathrm{Ca}^{2+}$ rhythms in the different clock neurons in vivo supports this view (Liang et al., 2016): $\mathrm{Ca}^{2+}$ rhythms in the l-LNvs peak in the middle of the day, unlike the s-LNvs, which display $\mathrm{Ca}^{2+}$ peaks in the late night/ early morning. We suggest that this phasing is produced by the inhibition of l-LNvs by the eyelets in the morning, followed by the excitation of the l-LNvs by the compound eyes and CRY. Interestingly, the only other clock neuron classes to display $\mathrm{Ca}^{2+}$ increases during the day are the LNds and fifth-sLNv, which phase lag the l-LNvs by $\sim 2.5 \mathrm{~h}$ and display peak $\mathrm{Ca}^{2+}$ levels in the late afternoon, a time that coincides with the evening peak of activity (Liang et al., 2016). We propose that the relative coordination of $\mathrm{Ca}^{2+}$ rhythms between the 1-LNvs and the LNds/fifth-sLNv is produced by the connection we have identified between these neurons and the action of the eyelet and visual system on the 1-LNvs (Fig. 6).

Recent work has revealed that evening activity is promoted directly by the evening oscillator neurons and that the mid-day siesta is produced by the daily inhibition of evening oscillators by a group of dorsal clock neurons (Guo et al., 2016). We propose that the connections we have described here govern the timing of the evening peak of activity through the PDF-dependent modulation of the molecular clocks within the evening oscillator neurons (Li et al., 2014) (Fig. 6, red arrows), although PDF modulation likely results in the excitation of target neurons (Seluzicki et al., 2014), which would promote evening activity. Our results reveal new and unexpected network properties underlying the entrainment of the circadian clock neuron network to LD cycles (summarized in Fig. 6). Excitatory effects of light on the LNvs are differentially modulated by the HB eyelets via cholinergic excitation of the s-LNvs and histaminergic inhibition of the l-LNvs. Our work further reveals PDF-dependent modulatory connections in the AMe between the l-LNvs and the s-LNvs and, most surprisingly, between the l-LNvs and a small subset of evening oscillators. Our work indicates that the latter connection is critical for the adjustment of evening activity phase during long, summer-like days. This network model of entrainment reveals not only how CRY and external photoreceptors interact within specific nodes of the CCNN, but also how photoreception is likely to drive changes in $\mathrm{CCNN}$ output in the face of changing day length.

\section{References}

Aschoff J (1981a) Biological rhythms. New York: Plenum.

Aschoff J (1981b) Freerunning and entrained circadian rhythms. In. New York: Plenum.

Baines RA, Uhler JP, Thompson A, Sweeney ST, Bate M (2001) Altered electrical properties in Drosophila neurons developing without synaptic transmission. J Neurosci 21:1523-1531. Medline

Barolo S, Carver LA, Posakony JW (2000) GFP and beta-galactosidase transformation vectors for promoter/enhancer analysis in Drosophila. Biotechniques 29:726-732. Medline

Bernstein JG, Garrity PA, Boyden ES (2012) Optogenetics and thermogenetics: technologies for controlling the activity of targeted cells within intact neural circuits. Curr Opin Neurobiol 22:61-71. CrossRef Medline

Burg MG, Sarthy PV, Koliantz G, Pak WL (1993) Genetic and molecular identification of a Drosophila histidine decarboxylase gene required in photoreceptor transmitter synthesis. EMBO J 12:911-919. Medline

Cao G, Nitabach MN (2008) Circadian control of membrane excitability in Drosophila melanogaster lateral ventral clock neurons. J Neurosci 28: 6493-6501. CrossRef Medline

Chen C, Buhl E, Xu M, Croset V, Rees JS, Lilley KS, Benton R, Hodge JJ, Stanewsky R (2015) Drosophila Ionotropic Receptor 25a mediates circadian clock resetting by temperature. Nature 527:516-520. CrossRef Medline

Chung BY, Kilman VL, Keath JR, Pitman JL, Allada R (2009) The GABAA receptor RDL acts in oeptidergic PDF neurons to promote sleep in Drosophila. Curr Biol 19:386-390. CrossRef Medline

Cusumano P, Klarsfeld A, Chélot E, Picot M, Richier B, Rouyer F (2009) PDF-modulated visual inputs and cryptochrome define diurnal behavior in Drosophila. Nat Neurosci 12:1431-1437. CrossRef Medline

Czeisler CA, Duffy JF, Shanahan TL, Brown EN, Mitchell JF, Rimmer DW, Ronda JM, Silva EJ, Allan JS, Emens JS, Dijk DJ, Kronauer RE (1999) Stability, precision, and near-24-hour period of the human circadian pacemaker. Science 284:2177-2181. CrossRef Medline

Díez-Noguera A (2013) Methods for serial analysis of long time series in the study of biological rhythms. J Circadian Rhythms 11:7. CrossRef Medline

Eck S, Helfrich-Förster C, Rieger D (2016) The timed depolarization of morning and evening oscillators phase shifts the circadian clock of Drosophila. J Biological Rhythms. Advance online publication. Retrieved Jun. 5, 2016. doi: 10.1177/0748730416651363. CrossRef Medline

Feinberg EH, Vanhoven MK, Bendesky A, Wang G, Fetter RD, Shen K, Bargmann CI (2008) GFP Reconstitution Across Synaptic Partners (GRASP) defines cell contacts and synapses in living nervous systems. Neuron 57: 353-363. CrossRef Medline

Fernández MP, Berni J, Ceriani MF (2008) Circadian remodeling of neuronal circuits involved in rhythmic behavior. PLoS Biol 6:e69. CrossRef Medline

Fogle KJ, Parson KG, Dahm NA, Holmes TC (2011) CRYPTOCHROME is a blue-light sensor that regulates neuronal firing rate. Science 331:1409_ 1413. CrossRef Medline

Glossop NR, Houl JH, Zheng H, Ng FS, Dudek SM, Hardin PE (2003) VRILLE feeds back to control circadian transcription of Clock in the Drosophila circadian oscillator. Neuron 37:249-261. CrossRef Medline

Golombek DA, Rosenstein RE (2010) Physiology of circadian entrainment. Physiol Rev 90:1063-1102. CrossRef Medline

Gordon MD, Scott K (2009) Motor control in a Drosophila taste circuit. Neuron 61:373-384. CrossRef Medline

Grima B, Chélot E, Xia R, Rouyer F (2004) Morning and evening peaks of 
activity rely on different clock neurons of the Drosophila brain. Nature 431:869-873. CrossRef Medline

Gummadova JO, Coutts GA, Glossop NR (2009) Analysis of the Drosophila Clock promoter reveals heterogeneity in expression between subgroups of central oscillator cells and identifies a novel enhancer region. J Biol Rhythms 24:353-367. CrossRef Medline

Guo F, Cerullo I, Chen X, Rosbash M (2014) PDF neuron firing phase-shifts key circadian activity neurons in Drosophila. Elife 3.

Guo F, Yu J, Jung HJ, Abruzzi KC, Luo W, Griffith LC, Rosbash M (2016) Circadian neuron feedback controls the Drosophila sleep-activity profile. Nature, in press.

Hamada FN, Rosenzweig M, Kang K, Pulver SR, Ghezzi A, Jegla TJ, Garrity PA (2008) An internal thermal sensor controlling temperature preference in Drosophila. Nature 454:217-220. CrossRef Medline

Hamasaka Y, Nässel DR (2006) Mapping of serotonin, dopamine, and histamine in relation to different clock neurons in the brain of Drosophila. J Comp Neurol 494:314-330. CrossRef Medline

Helfrich-Förster C (1997) Development of pigment-dispersing hormoneimmunoreactive neurons in the nervous system of Drosophila melanogaster. J Comp Neurol 380:335-354. CrossRef Medline

Helfrich-Förster C (1998) Robust circadian rhythmicity of Drosophila melanogaster requires the presence of lateral neurons: a brain-behavioral study of disconnected mutants. J Comp Physiol A 182:435-453. CrossRef Medline

Helfrich-Förster C (2002) The circadian system of Drosophila melanogaster and its light input pathways. Zoology (Jena) 105:297-312. CrossRef Medline

Helfrich-Förster C (2014) From neurogenetic studies in the fly brain to a concept in circadian biology. J Neurogenet 28:329-347. CrossRef Medline

Helfrich-Förster C, Winter C, Hofbauer A, Hall JC, Stanewsky R (2001) The circadian clock of fruit flies is blind after elimination of all known photoreceptors. Neuron 30:249-261. CrossRef Medline

Helfrich-Förster C, Edwards T, Yasuyama K, Wisotzki B, Schneuwly S, Stanewsky R, Meinertzhagen IA, Hofbauer A (2002) The extraretinal eyelet of Drosophila: development, ultrastructure, and putative circadian function. J Neurosci 22:9255-9266. Medline

Helfrich-Förster C, Yoshii T, Wülbeck C, Grieshaber E, Rieger D, Bachleitner W, Cusamano P, Rouyer F (2007) The lateral and dorsal neurons of Drosophila melanogaster: new insights about their morphology and function. Cold Spring Harb Symp Quant Biol 72:517-525. CrossRef Medline

Hermann-Luibl C, Yoshii T, Senthilan PR, Dircksen H, Helfrich-Förster C (2014) The ion transport peptide is a new functional clock neuropeptide in the fruit fly Drosophila melanogaster. J Neurosci 34:9522-9536. CrossRef Medline

Herzog ED (2007) Neurons and networks in daily rhythms. Nat Rev Neurosci 8:790-802. CrossRef Medline

Hewes RS, Park D, Gauthier SA, Schaefer AM, Taghert PH (2003) The bHLH protein Dimmed controls neuroendocrine cell differentiation in Drosophila. Development 130:1771-1781. CrossRef Medline

Hofbauer A, Buchner E (1989) Does Drosophila have seven eyes? Naturwissenschaften 76:335-336. CrossRef

Hong ST, Bang S, Paik D, Kang J, Hwang S, Jeon K, Chun B, Hyun S, Lee Y, Kim J (2006) Histamine and its receptors modulate temperaturepreference behaviors in Drosophila. J Neurosci 26:7245-7256. CrossRef Medline

Hyun S, Lee Y, Hong ST, Bang S, Paik D, Kang J, Shin J, Lee J, Jeon K, Hwang S, Bae E, Kim J (2005) Drosophila GPCR Han is a receptor for the circadian clock neuropeptide PDF. Neuron 48:267-278. CrossRef Medline

Im SH, Taghert PH (2010) PDF receptor expression reveals direct interactions between circadian oscillators in Drosophila. J Comp Neurol 518: 1925-1945. CrossRef Medline

Johard HA, Yoishii T, Dircksen H, Cusumano P, Rouyer F, Helfrich-Förster C, Nässel DR (2009) Peptidergic clock neurons in Drosophila: ion transport peptide and short neuropeptide F in subsets of dorsal and ventral lateral neurons. J Comp Neurol 516:59-73. CrossRef Medline

Lee G, Bahn JH, Park JH (2006) Sex- and clock-controlled expression of the neuropeptide F gene in Drosophila. Proc Natl Acad Sci U S A 103:1258012585. CrossRef Medline

Lelito KR, Shafer OT (2012) Reciprocal cholinergic and GABAergic modulation of the small ventrolateral pacemaker neurons of Drosophila's circadian clock neuron network. J Neurophysiol 107:2096-2108. CrossRef Medline

Liang X, Holy TE, Taghert PH (2016) Synchronous Drosophila circadian pacemakers display nonsynchronous $\mathrm{Ca}^{2+}$ rhythms in vivo. Science 351: 976-981. CrossRef Medline

Li Y, Guo F, Shen J, Rosbash M (2014) PDF and cAMP enhance PER stability in Drosophila clock neurons. Proc Natl Acad Sci U S A 111:E1284E1290. CrossRef Medline

Lima SQ, Miesenböck G (2005) Remote control of behavior through genetically targeted photostimulation of neurons. Cell 121:141-152. CrossRef Medline

Malpel S, Klarsfeld A, Rouyer F (2002) Larval optic nerve and adult extraretinal photoreceptors sequentially associate with clock neurons during Drosophila brain development. Development 129:1443-1453. Medline

McCarthy EV, Wu Y, Decarvalho T, Brandt C, Cao G, Nitabach MN (2011) Synchronized bilateral synaptic inputs to Drosophila melanogaster neuropeptidergic rest/arousal neurons. J Neurosci 31:8181-8193. CrossRef Medline

Menegazzi P, Vanin S, Yoshii T, Rieger D, Hermann C, Dusik V, Kyriacou CP, Helfrich-Förster C, Costa R (2013) Drosophila clock neurons under natural conditions. J Biol Rhythms 28:3-14. CrossRef Medline

Mertens I, Vandingenen A, Johnson EC, Shafer OT, Li W, Trigg JS, De Loof A, Schoofs L, Taghert PH (2005) PDF receptor signaling in Drosophila contributes to both circadian and geotactic behaviors. Neuron 48:213-219. CrossRef Medline

Münch M, Bromundt V (2012) Light and chronobiology: implications for health and disease. Dialogues Clin Neurosci 14:448-453. Medline

Muraro NI, Ceriani MF (2015) Acetylcholine from visual circuits modulates the activity of arousal neurons in Drosophila. J Neurosci 35:1631516327. CrossRef Medline

Nitabach MN, Taghert PH (2008) Organization of the Drosophila circadian control circuit. Curr Biol 18:R84-R93. CrossRef Medline

Pantazis A, Segaran A, Liu CH, Nikolaev A, Rister J, Thum AS, Roeder T, Semenov E, Juusola M, Hardie RC (2008) Distinct roles for two histamine receptors (hclA and hclB) at the Drosophila photoreceptor synapse. J Neurosci 28:7250-7259. CrossRef Medline

Parisky KM, Agosto J, Pulver SR, Shang Y, Kuklin E, Hodge JJ, Kang K, Liu X, Garrity PA, Rosbash M, Griffith LC (2008) PDF cells are a GABAresponsive wake-promoting component of the Drosophila sleep circuit. Neuron 60:672-682. CrossRef Medline

Petsakou A, Sapsis TP, Blau J (2015) Circadian rhythms in Rho1 activity regulate neuronal plasticity and network hierarchy. Cell 162:823-835. CrossRef Medline

Picot M, Cusumano P, Klarsfeld A, Ueda R, Rouyer F (2007) Light activates output from evening neurons and inhibits output from morning neurons in the Drosophila circadian clock. PLoS Biol 5:e315. CrossRef Medline

Pittendrigh CS (1981) Circadian systems: entrainment. In: Handbook of behavioral neurobiology (Aschoff J, ed), pp 95-124. New York: Plenum.

Pollack I, Hofbauer A (1991) Histamine-like immunoreactivity in the visual system and brain of Drosophila melanogaster. Cell Tissue Res 266: 391-398. CrossRef Medline

Potdar S, Sheeba V (2012) Large ventral lateral neurons determine the phase of evening activity peak across photoperiods in Drosophila melanogaster. J Biol Rhythms 27:267-279. CrossRef Medline

Renn SC, Park JH, Rosbash M, Hall JC, Taghert PH (1999) Apdf neuropeptide gene mutation and ablation of PDF neurons each cause severe abnormalities of behavioral circadian rhythms in Drosophila. Cell 99:791-802. CrossRef Medline

Rieger D, Stanewsky R, Helfrich-Förster C (2003) Cryptochrome, compound eyes, Hofbauer-Buchner eyelets, and ocelli play different roles in the entrainment and masking pathway of the locomotor activity rhythm in the fruit fly Drosophila melanogaster. J Biol Rhythms 18:377-391. CrossRef Medline

Rieger D, Shafer OT, Tomioka K, Helfrich-Förster C (2006) Functional analysis of circadian pacemaker neurons in Drosophila melanogaster. J Neurosci 26:2531-2543. CrossRef Medline

Roenneberg T, Merrow M (2003) The network of time: understanding the molecular circadian system. Curr Biol 13:R198-R207. CrossRef Medline

Ruben M, Drapeau MD, Mizrak D, Blau J (2012) A mechanism for circadian control of pacemaker neuron excitability. J Biol Rhythms 27:353-364. CrossRef Medline

Schlichting M, Helfrich-Förster C (2015) Photic entrainment in Drosophila assessed by locomotor activity recordings. Methods Enzymol 552: 105-123. CrossRef Medline

Schmid B, Helfrich-Förster C, Yoshii T (2011) A new ImageJ plug-in 
"ActogramJ" for chronobiological analyses. J Biol Rhythms 26:464467. CrossRef Medline

Seluzicki A, Flourakis M, Kula-Eversole E, Zhang L, Kilman V, Allada R (2014) Dual PDF signaling pathways reset clocks via TIMELESS and acutely excite target neurons to control circadian behavior. PLOS Biol 12:e1001810. CrossRef Medline

Shafer OT, Taghert PH (2009) RNA-interference knockdown of Drosophila pigment dispersing factor in neuronal subsets: the anatomical basis of a neuropeptide's circadian functions. PLoS One 4:e8298. CrossRef Medline

Shafer OT, Kim DJ, Dunbar-Yaffe R, Nikolaev VO, Lohse MJ, Taghert PH (2008) Widespread receptivity to neuropeptide PDF throughout the neuronal circadian clock network of Drosophila revealed by real-time cyclic AMP imaging. Neuron 58:223-237. CrossRef Medline

Shang Y, Griffith LC, Rosbash M (2008) Light-arousal and circadian photoreception circuits intersect at the large PDF cells of the Drosophila brain. Proc Natl Acad Sci U S A 105:19587-19594. CrossRef Medline

Sheeba V, Gu H, Sharma VK, O’Dowd DK, Holmes TC (2008) Circadianand light-dependent regulation of resting membrane potential and spontaneous action potential firing of Drosophila circadian pacemaker neurons. J Neurophysiol 99:976-988. CrossRef Medline

Sheeba V, Fogle KJ, Holmes TC (2010) Persistence of morning anticipation behavior and high amplitude morning startle response following functional loss of small ventral lateral neurons in Drosophila. PLoS One 5:e11628. CrossRef Medline

Siegmund T, Korge G (2001) Innervation of the ring gland of Drosophila melanogaster. Comp Neurol 431:481-491. CrossRef Medline

Sivachenko A, Li Y, Abruzzi KC, Rosbash M (2013) The transcription factor Mef2 links the Drosophila core clock to Fas2, neuronal morphology, and circadian behavior. Neuron 79:281-292. CrossRef Medline

Sprecher SG, Desplan C (2008) Switch of rhodopsin expression in terminally differentiated Drosophila sensory neurons. Nature 454:533-537. CrossRef Medline

Stewart BA, Atwood HL, Renger JJ, Wang J, Wu CF (1994) Improved stability of Drosophila larval neuromuscular preparations in haemolymphlike physiological solutions. J Comp Physiol A 175:179-191. CrossRef Medline

Tian L, Hires SA, Mao T, Huber D, Chiappe ME, Chalasani SH, Petreanu L, Akerboom J, McKinney SA, Schreiter ER, Bargmann CI, Jayaraman V, Svoboda K, Looger LL (2009) Imaging neural activity in worms, flies and mice with improved GCaMP calcium indicators. Nat Methods 6:875881. CrossRef Medline

Veleri S, Rieger D, Helfrich-Förster C, Stanewsky R (2007) HofbauerBuchner eyelet affects circadian photosensitivity and coordinates TIM and PER expression in Drosophila clock neurons. J Biol Rhythms 22: 29-42. CrossRef Medline

Viswanath V, Story GM, Peier AM, Petrus MJ, Lee VM, Hwang SW, Patapoutian A, Jegla T (2003) Opposite thermosensor in fruitfly and mouse. Nature 423:822-823. CrossRef Medline

Welsh DK, Takahashi JS, Kay SA (2010) Suprachiasmatic nucleus: cell autonomy and network properties. Annu Rev Physiol 72:551-577. CrossRef Medline

Wülbeck C, Grieshaber E, Helfrich-Förster C (2009) Blocking endocytosis in Drosophila's circadian pacemaker neurons interferes with the endogenous clock in a PDF-dependent way. Chronobiol Int 26:1307-1322. CrossRef Medline

Yao Z, Shafer OT (2014) The Drosophila circadian clock is a variably coupled network of multiple peptidergic units. Science 343:1516-1520. CrossRef Medline

Yao Z, Macara AM, Lelito KR, Minosyan TY, Shafer OT (2012) Analysis of functional neuronal connectivity in the Drosophila brain. J Neurophysiol 108:684-696. CrossRef Medline

Yasuyama K, Meinertzhagen IA (1999) Extraretinal photoreceptors at the compound eye's posterior margin in Drosophila melanogaster. J Comp Neurol 412:193-202. CrossRef Medline

Yasuyama K, Salvaterra PM (1999) Localization of choline acetyltransferase-expressing neurons in Drosophila nervous system. Microsc Res Tech 45:65-79. CrossRef Medline

Yoshii T, Todo T, Wülbeck C, Stanewsky R, Helfrich-Förster C (2008) Cryptochrome is present in the compound eyes and a subset of Drosophila's clock neurons. J Comp Neurol 508:952-966. CrossRef Medline

Yoshii T, Wülbeck C, Sehadova H, Veleri S, Bichler D, Stanewsky R, HelfrichFörster C (2009) The neuropeptide pigment-dispersing factor adjusts period and phase of Drosophila's clock. J Neurosci 29:2597-2610. CrossRef Medline

Zhang L, Chung BY, Lear BC, Kilman VL, Liu Y, Mahesh G, Meissner RA, Hardin PE, Allada R (2010) DN1(p) circadian neurons coordinate acute light and PDF inputs to produce robust daily behavior in Drosophila. Curr Biol 20:591-599. CrossRef Medline 